\title{
Infinitely Many Coexisting Attractors in No-Equilibrium Chaotic System
}

\author{
Qiang Lai $\mathbb{D}^{1},{ }^{1}$ Paul Didier Kamdem Kuate $\mathbb{D}^{2},{ }^{2}$ Huiqin Pei $\mathbb{D},{ }^{1}$ and Hilaire Fotsin $\mathbb{D}^{2}$ \\ ${ }^{1}$ School of Electrical and Automation Engineering, East China Jiaotong University, Nanchang 330013, China \\ ${ }^{2}$ Laboratory of Condensed Matter, Electronics and Signal Processing Department of Physics, University of Dschang, P.O. Box 067, \\ Dschang, Cameroon \\ Correspondence should be addressed to Qiang Lai; laiqiang87@126.com
}

Received 17 January 2020; Revised 18 February 2020; Accepted 26 February 2020; Published 28 March 2020

Guest Editor: Chun-Lai Li

Copyright (C) 2020 Qiang Lai et al. This is an open access article distributed under the Creative Commons Attribution License, which permits unrestricted use, distribution, and reproduction in any medium, provided the original work is properly cited.

This paper proposes a new no-equilibrium chaotic system that has the ability to yield infinitely many coexisting hidden attractors. Dynamic behaviors of the system with respect to the parameters and initial conditions are numerically studied. It shows that the system has chaotic, quasiperiodic, and periodic motions for different parameters and coexists with a large number of hidden attractors for different initial conditions. The circuit and microcontroller implementations of the system are given for illustrating its physical meaning. Also, the synchronization conditions of the system are established based on the adaptive control method.

\section{Introduction}

Encouraging progress has been made on chaos in the past few decades. An important change is to recognize the great application potential of chaos in engineering. Nowadays, chaos generation has become an important research issue arousing constant concern. Inspired by the well-known Lorenz system [1], many different chaotic systems have been created [2-7]. There are two interesting directions in generating new chaotic systems. One is to discover chaos in nonlinear systems with very simple mathematical models. The most representative work was made by Sprott who established nineteen polynomial chaotic systems with either five terms and two nonlinear terms or six terms and one nonlinear term [8]. The other is to construct chaotic systems with special strange attractors including butterfly attractor, multiscroll attractor, multiwing attractor, hidden attractor, and coexisting attractors [9-14]. The number and type of equilibria play a decisive role in the dynamic properties of chaotic system to some extent. A classic argument is that a dynamic system with one saddle focus connected by homoclinic orbit or two saddle foci connected by heteroclinic orbit generates horseshoe chaos [15]. It is generally recognized that no equilibrium chaotic system may generate hidden attractor. Also, a lot of previous studies have shown that chaotic systems with multiple unstable equilibria usually have richer dynamic behaviors and are more likely to produce coexisting attractors. Therefore, many scholars tend to construct chaotic systems and distinguish their dynamic properties by configuring different quantities and types of equilibria. Chaotic systems with no equilibrium, one stable equilibrium, one unstable node, two saddle foci, circular equilibria, a line equilibria, and other types of equilibria have been reported [16-19].

Recently, the study of coexisting attractors and hidden attractors in chaotic systems has aroused great enthusiasm among scholars. Li et al. studied the coexisting attractors in Lorenz systems by some numerical experiments and proposed the conditional symmetry method to yield any number of coexisting attractors [20,21]. Kengne et al. put forward some simple jerk systems with coexisting attractors [22]. Lai et al. introduced some effective methods to construct chaotic systems with infinitely many coexisting strange attractors [23,24]. Li et al. designed a programmable chaotic circuit with infinitely many chaotic attractors and established the coexistence of multiple attractors by simulation methods [25]. Bao et al. found that memristor-based 
chaotic systems can generate different types of coexisting attractors [26, 27]. Li et al. studied the coexisting attractors in memristor-based chaotic circuit in depth and considered its application in image encryption [28, 29]. Wei et al. studied the nonstationary chimeras in Hindmarsh-Rose neuronal network and found that the network coexists with periodic and chaotic states [30]. The history of studying the hidden attractors in dynamical systems without equilibria dates back to the well-known Sommerfeld effect, arising from feedback in the energy exchange between vibrating systems [31]. The electromechanical and drilling systems without equilibria have the ability to yield multiple hidden attractors $[32,33]$. It is very interesting to generate as many hidden attractors as possible in chaotic system via some periodic functions which can continuously replicate the attractors in phase space. The attractors produced by this method usually have the same properties. Another interesting question is how many nontrivial attractors can be coexist in phase space of nonlinear system, which corresponds to a chaotic generalization [34] of the second part of Hilbert's 16th problem. Recently, the study of hidden attractors in chaotic systems has received increasing concern. Wei et al. generated an extended Rikitake system with hidden attractors [35] and investigated the hidden hyperchaos of five-dimensional self-exciting homopolar disc dynamo via analytical and numerical approaches [36]. Pham et al. did many important works on hidden attractors and first showed different families of hidden attractors in chaotic systems [37, 38]. Danca et al. discovered the hidden attractors of the classic Rabinovich-Fabrikant system [39]. Hidden attractor that refers to attractor whose basin of attraction does not intersect with small neighborhoods of equilibria [40] embodies some mysterious unknown dynamic behaviors of the system. The phenomenon of coexisting attractors that corresponds to the generation of multiple attractors with independent basins of attractions implies the strong influence of initial conditions on the final state of system. Both hidden attractors and coexisting attractors are interesting nonlinear dynamics worthy of further study. The construction of simple no-equilibrium chaotic system with an infinite number of hidden attractors is an interesting and challenging thing. It will be of great significance to reveal the complex behaviors of simple systems. However, most existing systems can generate a limited number of attractors. So this paper aims to present a new autonomous chaotic system with infinitely many coexisting hidden attractors from the perspective of building complex behavior of simple system. Necessary theoretical and experimental researches are given to illustrate the dynamic properties of the proposed system. The circuit and microcontroller implementations and synchronization control of the system are studied. The paper is organized as follows. Section 2 derives the model of the new system. Section 3 presents the coexisting hidden attractors of the system. Section 4 implements the system for physically illustrating its dynamics. Section 5 considers its synchronization problem. Section 6 summarizes the conclusions.

\section{New Chaotic System}

Looking back, an augmented Sprott B system is described by the following differential equations [41]:

$$
\left\{\begin{array}{l}
\dot{x}_{1}=a\left(x_{2}-x_{1}\right), \\
\dot{x}_{2}=x_{1} x_{3}+x_{4}, \\
\dot{x}_{3}=b-x_{1} x_{2}, \\
\dot{x}_{4}=-c x_{2},
\end{array}\right.
$$

where $x_{1}, x_{2}, x_{3}$, and $x_{4}$ are state variables and $a, b$, and $c$ are parameters. The previous study has shown that system (1) has no equilibrium and coexists with two symmetric strange attractors. Replacing the nonlinear term $x_{1} x_{3}$ of system (1) with $k x_{1} \sin \left(x_{3}\right)(k>0$ is a real number), then the following new chaotic system is established:

$$
\left\{\begin{array}{l}
\dot{x}_{1}=a\left(x_{2}-x_{1}\right), \\
\dot{x}_{2}=k x_{1} \sin \left(x_{3}\right)+x_{4}, \\
\dot{x}_{3}=b-x_{1} x_{2}, \\
\dot{x}_{4}=-c x_{2} .
\end{array}\right.
$$

System (2) and system (1) have some similar properties including the following: (i) they have no equilibrium because there exists no point that satisfies $\dot{x}_{1}=\dot{x}_{2}=\dot{x}_{3}=\dot{x}_{4}=0$; (ii) they are dissipative with the divergence $\nabla V=\partial \dot{x}_{1} / \partial x_{1}+$ $\partial \dot{x}_{2} / \partial x_{2}+\partial \dot{x}_{3} / \partial x_{3}+\partial \dot{x}_{4} / \partial x_{4}=-a<0$ for $a>0$; (iii) they have the symmetry under the transformation $\left(x_{1}, x_{2}\right.$, $\left.x_{3}, x_{4}\right) \longrightarrow\left(-x_{1},-x_{2}, x_{3},-x_{4}\right)$; and (iv) they have hidden attractors whose basins of attraction do not intersect with small neighborhoods of any equilibria. However, the remarkable difference between them is that system (2) can generate infinitely many coexisting hidden attractors while system (1) only generates no more than three attractors for given parameters. Also, to our best knowledge, system (2) has never been reported before. Thus, system (2) can be classified as a new chaotic system. The following section will give an intuitive observation of the dynamic properties of system (2).

\section{Coexisting Hidden Attractors}

Here, we will investigate the influence of the parameters and initial conditions on the dynamic behaviors of system (2) by simulation experiments, emphasizing the existence of infinitely many hidden chaotic and periodic attractors in system (2). We apply the well-known fourth-fifth-order Runge-Kutta method to numerically solved system (2) on Matlab platform. All the simulation results are obtained by fixing the step size $\Delta t=0.01$ and time region $t \in[0,300]$. The Lyapunov exponents are calculated along the trajectories of system (2) by using the Wolf method [42]. We also can further consider the Lyapunov exponents by using the method proposed in literature [43]. Here, the Lyapunov exponents are used to distinguish the chaotic, periodic, and stable states of system (2). So, we use the conventional method to calculate them. There is no doubt that a system with the positive largest Lyapunov exponent cannot be 
judged to be chaotic directly. It also requires that the system is bounded. However, it is difficult to quantitatively establish the boundedness of the system by using the analytical method. In our simulations, we try many experiments to demonstrate the boundedness of system (2). It shows that all the state variables remain in a bounded range for a long enough time in our simulations, which partially implies the boundedness of system (2). Actually, the dissipativity of system (2) for $a>0$ can partially determine its boundedness. Also, the literature [44] gives an interesting work corresponding to a new idea on the boundedness of chaotic systems.

Firstly, we study the dynamic evolution of system (2) with respect to the parameters. It is easy to generate the bifurcation diagram and Lyapunov exponents of system (2) for the parameters $a \in[4,20], b=c=1$, and $k=12$ and initial value $(1,1,1,1)$. From Figure 1, we get that system (2) makes chaotic, quasiperiodic, and periodic motions for different values of $a$. When $a=4$, system (2) has a strange attractor shown in Figure 2. The positive maximum Lyapunov exponent $L_{1} \approx 0.4456$ and fractal Lyapunov dimension $D_{L} \approx 3.0996$ imply the chaotic feature of the attractor. When $a=12$, system (2) performs quasiperiodic motion as its first two Lyapunov exponents $L_{1}=L_{2} \approx 0$. The phase portraits of the quasiperiodic attractor are given in Figure 3. When $a=20$, system (2) performs periodic motion as illustrated in Figure 4.

We also can illustrate the dynamic evolution of system (2) corresponding to the parameters $a=4, b=1, c \in[1,12]$, and $k=12$ and initial value $(1,1,1,1)$ via the bifurcation diagram and Lyapunov exponents in Figure 5. It indicates that the chaotic, quasiperiodic, and periodic motions will generate with the variation of $c$. Figure 6 shows the chaotic and quasiperiodic motions of system (2) with $c=6,12$.

Secondly, we will consider the influence of initial value on the final state of system (2). Let the parameters $a=4$, $b=c=1$, and $k=12$ and initial value $x_{0}=\left(x_{10}, x_{20}, x_{30}\right.$, $x_{40}$ ) with $x_{10}=x_{20}=x_{40}=1$; then we can plot the bifurcation diagram and Lyapunov exponents of system (2) for $x_{30} \in[0,35]$, as given in Figure 7 . It is easy to know that system (2) coexists with six chaotic attractors for $x_{30} \in[0,35]$ from Figure 7 . We can obtain the attractors by plotting the phase portraits of system (2) from initial values $(1,1,2 j \pi, 1), j=0,1,2,, 3,4,5$. As shown in Figure 8, the attractors are generated along the $x_{3}$-axis and all these attractors have the same nature. Also, the coexisting quasiperiodic (or periodic) attractors can be obtained in system (2) by fixing $a=12, b=c=1$, and $k=12$ (or $a=20$, $b=c=1$, and $k=12$ ). Figure 9 shows six quasiperiodic (or periodic) attractors of system (2) yielded from initial values $(1,1,2 j \pi, 1), j=0,1,2,3,4,5$. As a matter of fact, system (2) has the ability to generate infinitely many hidden attractors from initial values $(1,1,2 j \pi, 1), j=0, \pm 1, \pm 2, \ldots, \pm n$ along the $x_{3}$-axis.

\section{Circuit and Microcontroller Realization}

From the theoretical study presented above, it is predicted that system (2) exhibits a plethora of interesting dynamic behaviors for suitable sets of parameters and initial conditions. The goal of this section is to investigate the practical implementation of system (2). The corresponding circuit in Figure 10 is designed using commercially available off-theshelf electronic components, operational amplifiers, and analog multipliers AD633 integrated circuits, all powered by $\pm 18 \mathrm{~V}$ symmetric voltages.

The state variables $x_{1}, x_{2}, x_{3}$, and $x_{4}$ are emulated by output signals of integrators. Using the basic electrical circuit's laws, system (2) can be rewritten as

$$
\left\{\begin{array}{l}
x_{1}=\frac{R_{4}}{R_{1}} \frac{1}{R C_{1}} \int\left(\frac{R}{R_{2}} x_{2}-\frac{R}{R_{1}} x_{1}\right) \mathrm{d} t, \\
x_{2}=\frac{R_{8}}{R_{7}} \frac{1}{R C_{2}} \int\left(\frac{R}{R_{5}} x_{1} \sin \left(x_{3}\right)-\frac{R}{R_{6}} x_{4}\right) \mathrm{d} t, \\
x_{3}=\frac{R_{12}}{R_{11}} \frac{1}{R C_{3}} \int\left(\frac{R}{R_{10}} V_{b}-\frac{R}{R_{9}} x_{1} x_{2}\right) \mathrm{d} t, \\
x_{4}=\frac{1}{R C_{4}} \int\left(\frac{R}{R_{13}} x_{2}\right) \mathrm{d} t .
\end{array}\right.
$$

By taking $C_{1}=C_{2}=C_{3}=C_{4}=C, R_{6}=R_{9}=R_{10}=R$, $R_{3}=R_{4}=R_{7}=R_{8}=R_{11}=R_{12}=10 \mathrm{k} \Omega$, and $R_{1}=R_{2}=R_{a}$, we have the parameters $a=R / R_{a}, k=R / R_{5}, b=V_{b}$, and $c=$ $R / R_{13}$ and the time scaling factor $\tau=t / R C$. From this point, it is easy to demonstrate that the differentiation of system (3) is equivalent to the original system (2). Let $C=10 \mathrm{nF}$, $R=100 \mathrm{k} \Omega, R_{a}=25 \mathrm{k} \Omega, R_{5}=8.3333 \mathrm{k} \Omega, R_{13}=100 \mathrm{k} \Omega$, and $V_{b}=1 \mathrm{~V}$; a chaotic behavior is observed in Figure 11. Periodic and quasiperiodic orbits are also noticed from the analog circuit; see Figure 12. Other cases of chaotic and quasiperiodic behaviors are also observed from the analog circuit, as illustrated in Figures 13 and 14.

Up to five coexisting chaotic attractors, five coexisting limit cycles, and five coexisting quasiperiodic orbits are noticed for the initial voltage of the capacitor $C_{3}$ successively fixed as $(1,1,-6 \pi), \quad(1,1,-4 \pi), \quad(1,1,-2 \pi), \quad(1,1,0)$, $(1,1,2 \pi)$, and $(1,1,4 \pi)$ as illustrated in Figure 15 .

Next, we will present the microcontroller-based implementation of system (2) by using an Arduino Mega 2650 board. It is a low-cost open-source and easy-to-use electronic board based on the ATMEGA2560 microcontroller. The designed system is simulated and realized using the scheme on Figure 16. The digital outputs of the microcontroller board are connected to two R-2R ladders acting here as Digital-to-Analog Converters (DAC). The converted analog signals corresponding to the state variables of system (2) are simultaneously visualized on an oscilloscope and a computer through a low-cost data acquisition module. The program is written in such a way that the magnitude of the output signals can be adjusted within the voltage range $0-5 \mathrm{~V}$. Figure 17 shows the practical setup of the experiment on the microcontroller-based implementation of system (2). 

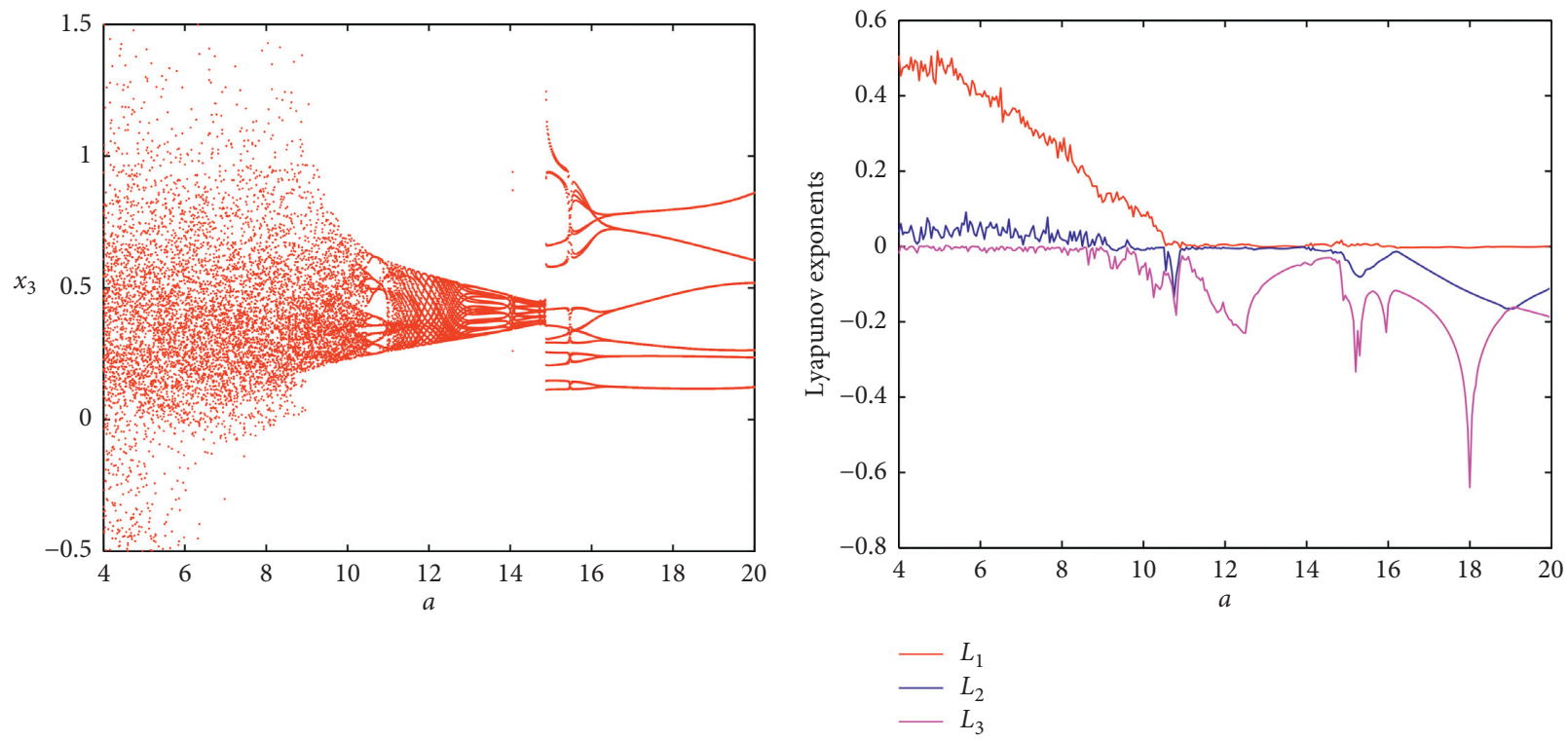

(a)

(b)

FIgURe 1: Bifurcation diagram and Lyapunov exponent of system (2) versus $a \in[4,20]$ from initial value $(1,1,1,1)$, where $L_{1}>L_{2}>L_{3}>L_{4}$ are Lyapunov exponents of system (2) and $L_{4}$ is always less than -4 .

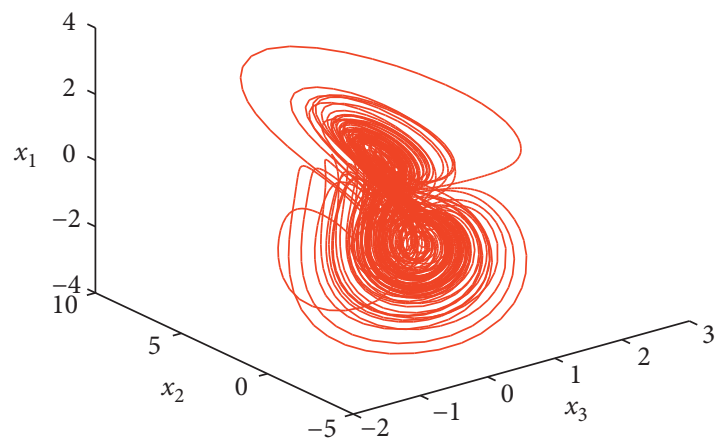

(a)

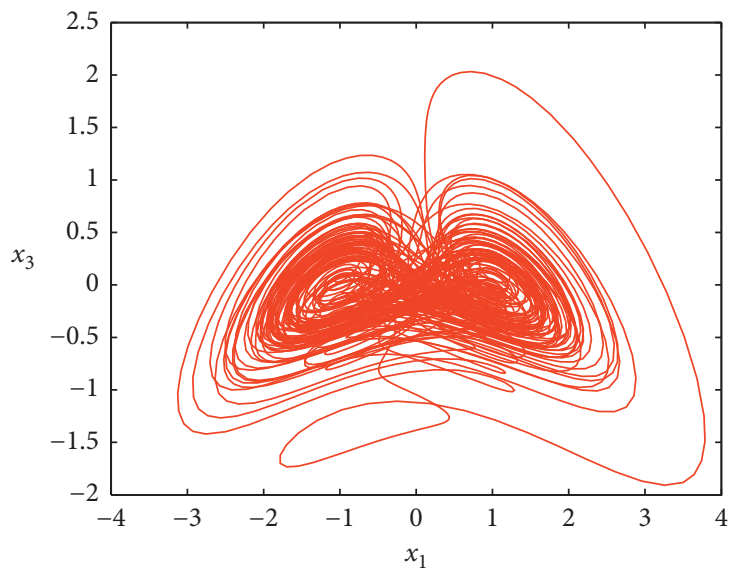

(c)

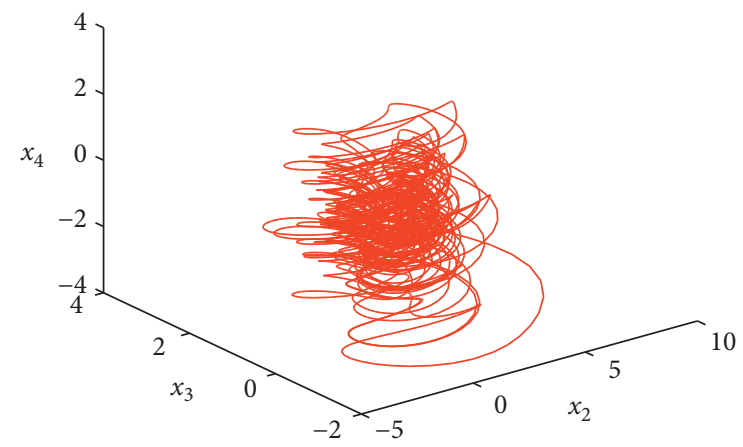

(b)

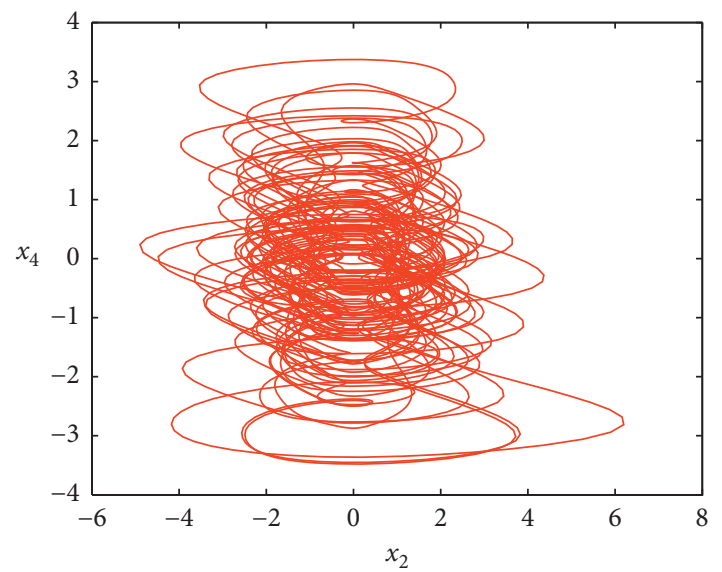

(d)

FIGURE 2: Chaotic attractor of system (2) with $a=4, b=c=1$, and $k=12$ : (a) $x_{3}-x_{2}-x_{1}$; (b) $x_{2}-x_{3}-x_{4}$; (c) $x_{1}-x_{3}$; (d) $x_{2}-x_{4}$. 


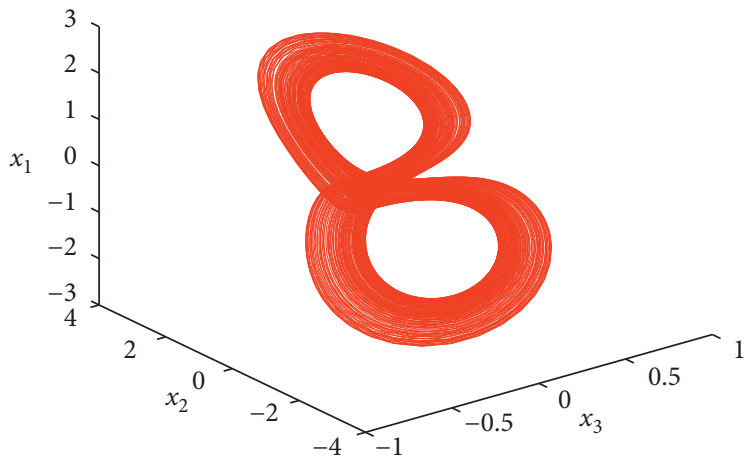

(a)

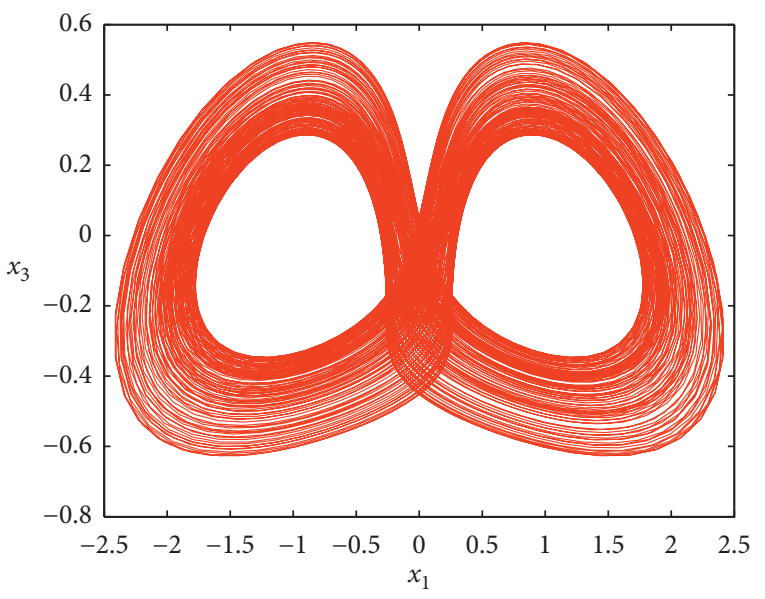

(c)

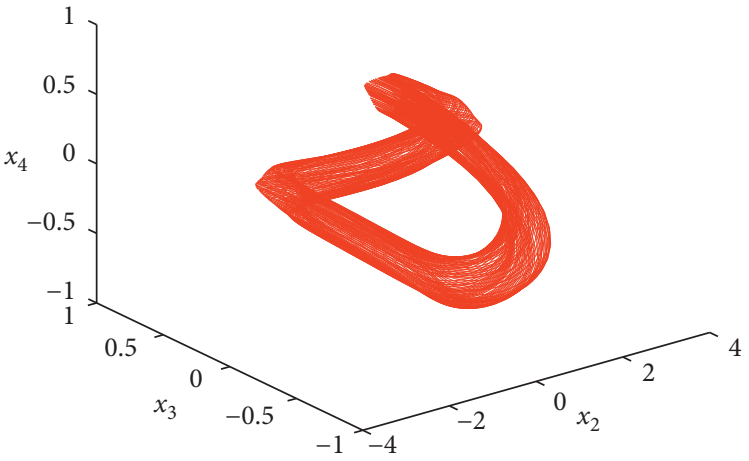

(b)

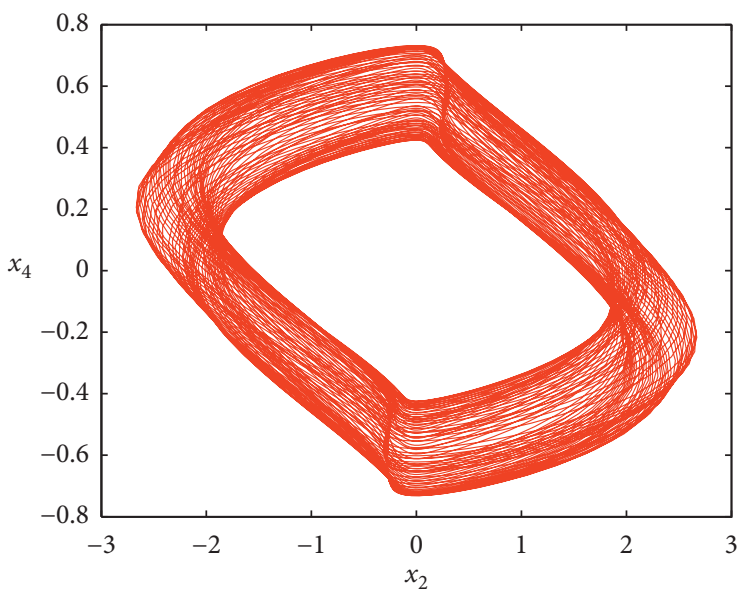

(d)

Figure 3: Quasiperiodic motion of system (2) with $a=12, b=c=1$, and $k=12$ : (a) $x_{3}-x_{2}-x_{1}$; (b) $x_{2}-x_{3}-x_{4}$; (c) $x_{1}-x_{3}$; (d) $x_{2}-x_{4}$.

The phase portraits and time series obtained from the microcontroller-based implementation of system (2) confirm all the predicted dynamic behaviors. Chaotic, periodic, and quasiperiodic motions are all observed as illustrated in Figure 18. Other cases of chaotic and quasiperiodic motions are also observed for appropriated sets of parameters, as illustrated in Figure 19.

The phase portraits obtained from the microcontrollerbased implementation, the analog circuit, and the numerical study are all in accordance. The new chaotic system is able to generate a multitude of coexisting attractors under various types of dynamic behaviors.

\section{Synchronization Control}

In various chaos-based technological applications like secure communication or cryptography, synchronization is needed between a master system (sender) and a slave system (receiver). The practical realization of such mechanisms is subject to many challenges. In electronic implementations, the ageing, the temperature effects, the electromagnetic activity of the environment, and the nonideal behavior of the channel are some of the various factors which can induce huge errors and undesirable consequences. This is why it is important to design an adaptive synchronization scheme for chaotic system with unknown parameters. The adaptive synchronization of chaotic system has important practical significance. It extends the engineering applications of chaotic system and shows the ability of chaotic system to change its own characteristics or use the external control input to achieve synchronization when the information of the system and environment has some uncertainty. Among the several synchronization techniques, the adaptive method appears to be one of the most interesting because of its robustness and its simplicity of implementation. It has proved to be an effective method to synchronize the chaotic system with unknown parameters $[45,46]$. In many practical systems, the parameters of systems are probably unknown or change from time to time. For such systems, the adaptive control is a better choice to realize the synchronization. Also, the adaptive control can be used in combination with other control methods. In addition, no prior knowledge of parameters is needed for master system and slave system to be synchronized [47, 48]. Because of these advantages, the adaptive control is widely accepted in academic circles and its application on synchronization has been reported by many scholars $[49,50]$. This section will consider the adaptive synchronization problem of system (2). The result is based on the Lyapunov stability theory. All the parameters of the master system are accessible to measurements and those of the slave system are unknown. Consider the following master system: 


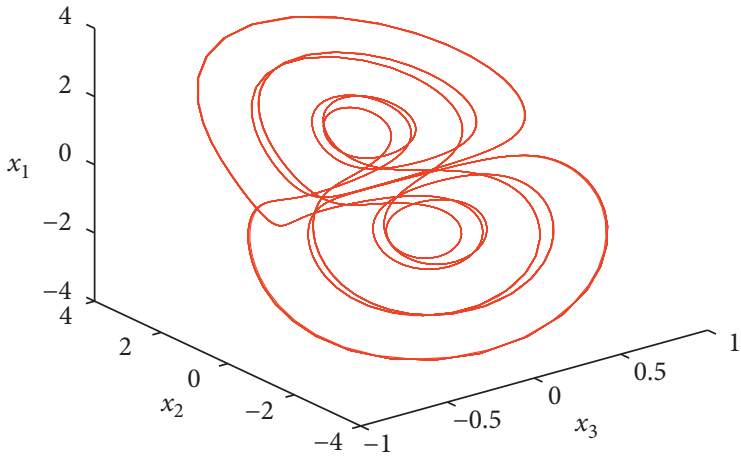

(a)

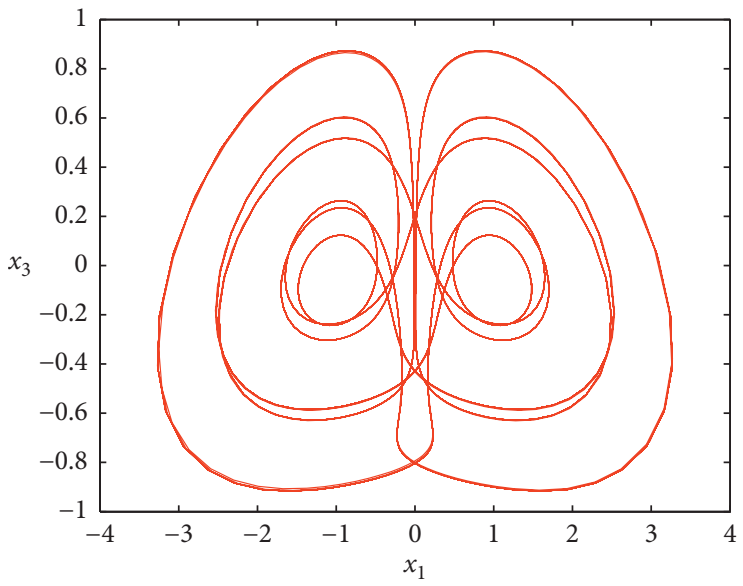

(c)

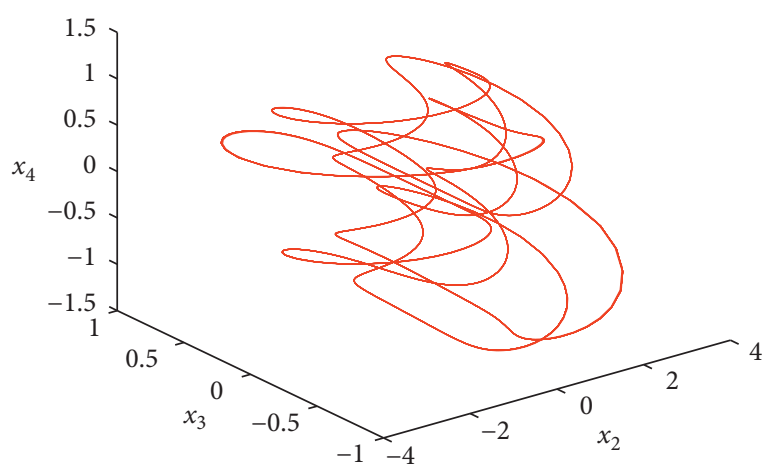

(b)

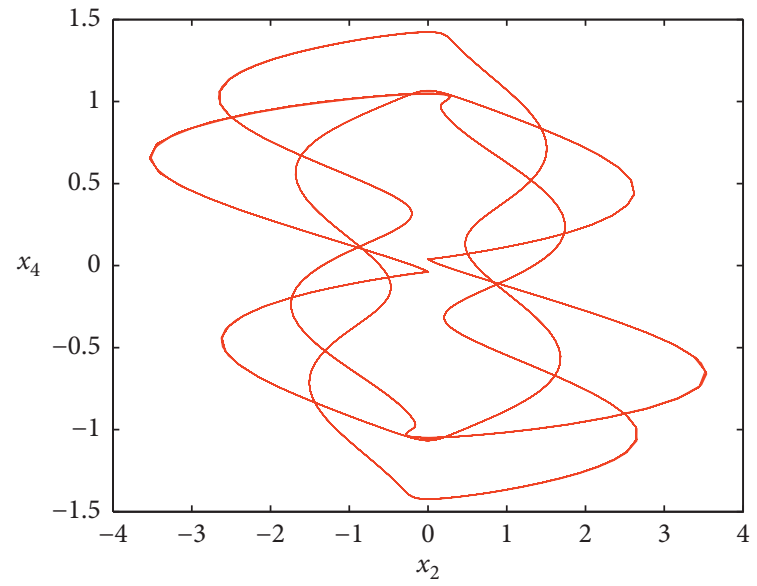

(d)

Figure 4: Periodic motion of system (2) with $a=20, b=c=1$, and $k=12$ : (a) $x_{3}-x_{2}-x_{1}$; (b) $x_{2}-x_{3}-x_{4}$; (c) $x_{1}-x_{3}$; (d) $x_{2}-x_{4}$.
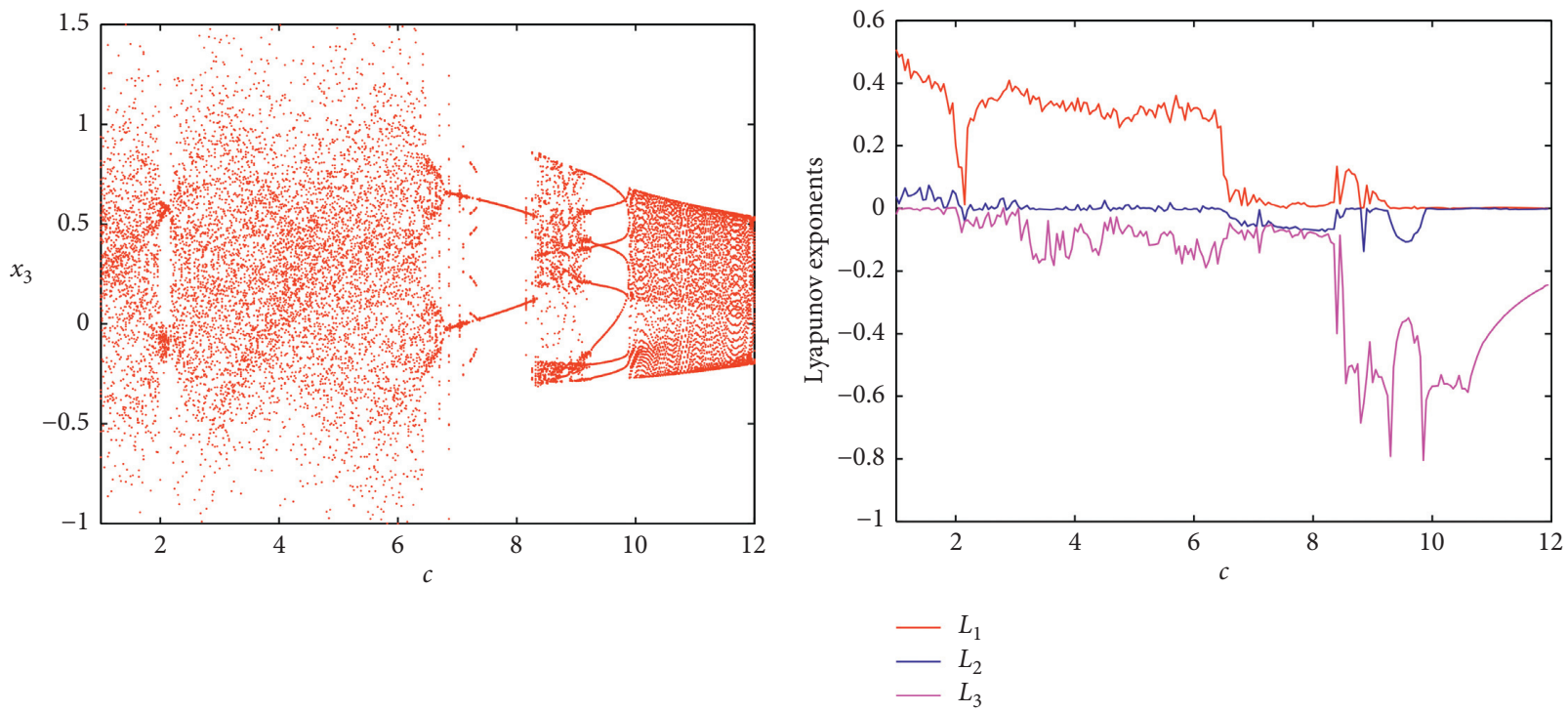

(a)

(b)

FIgURe 5: Bifurcation diagram and Lyapunov exponents of system (2) versus $c \in[1,12]$ from initial value $(1,1,1,1)$, where $L_{1}>L_{2}>L_{3}>L_{4}$ are Lyapunov exponents of system (2) and $L_{4}$ is always less than -4 . 


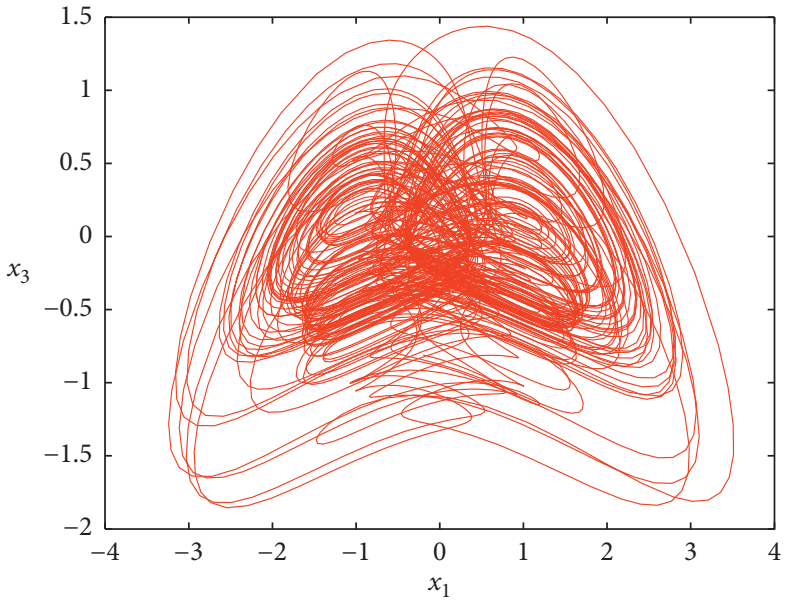

(a)

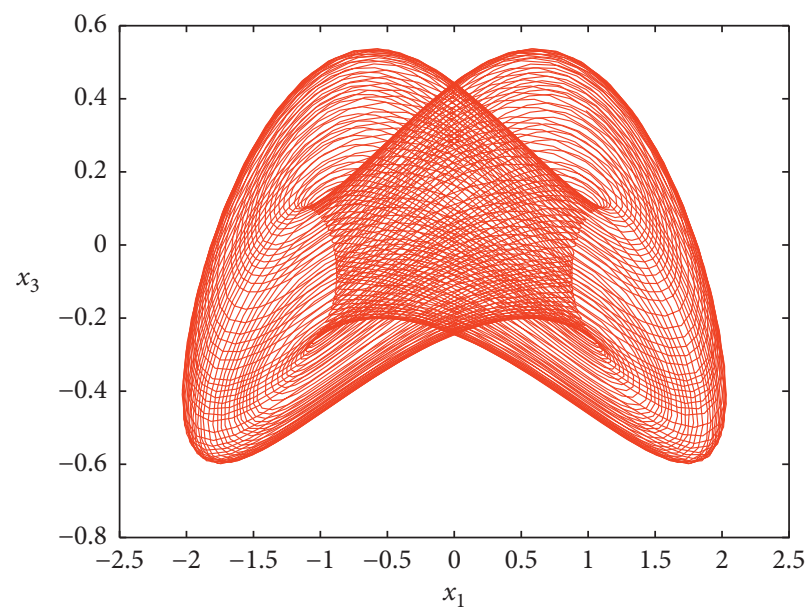

(c)

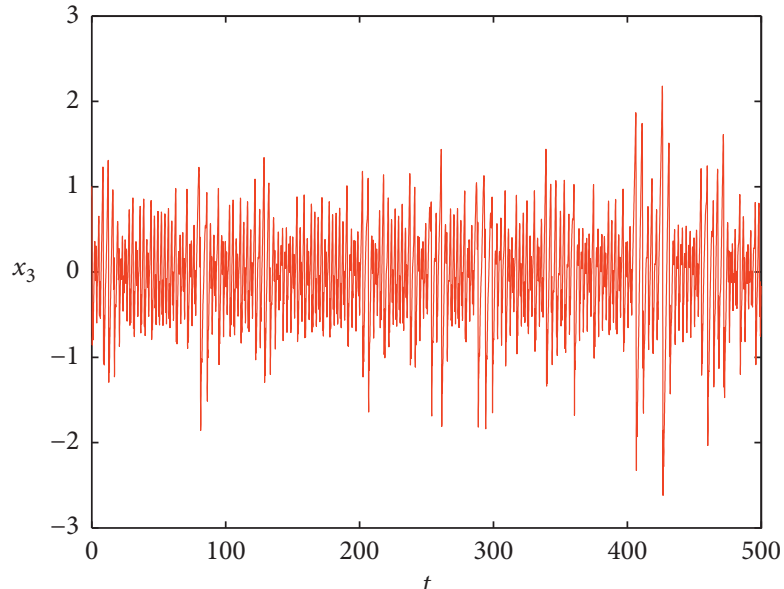

(b)

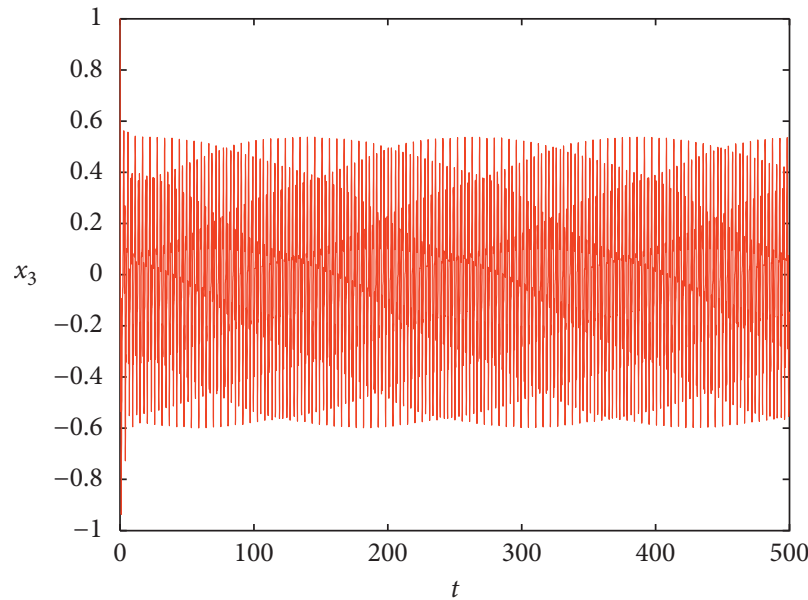

(d)

FIGURE 6: Chaotic and quasiperiodic motions of system (2) with $a=4$ and $b=1:(\mathrm{a}, \mathrm{b}) c=6$; (c, d) $c=12$.
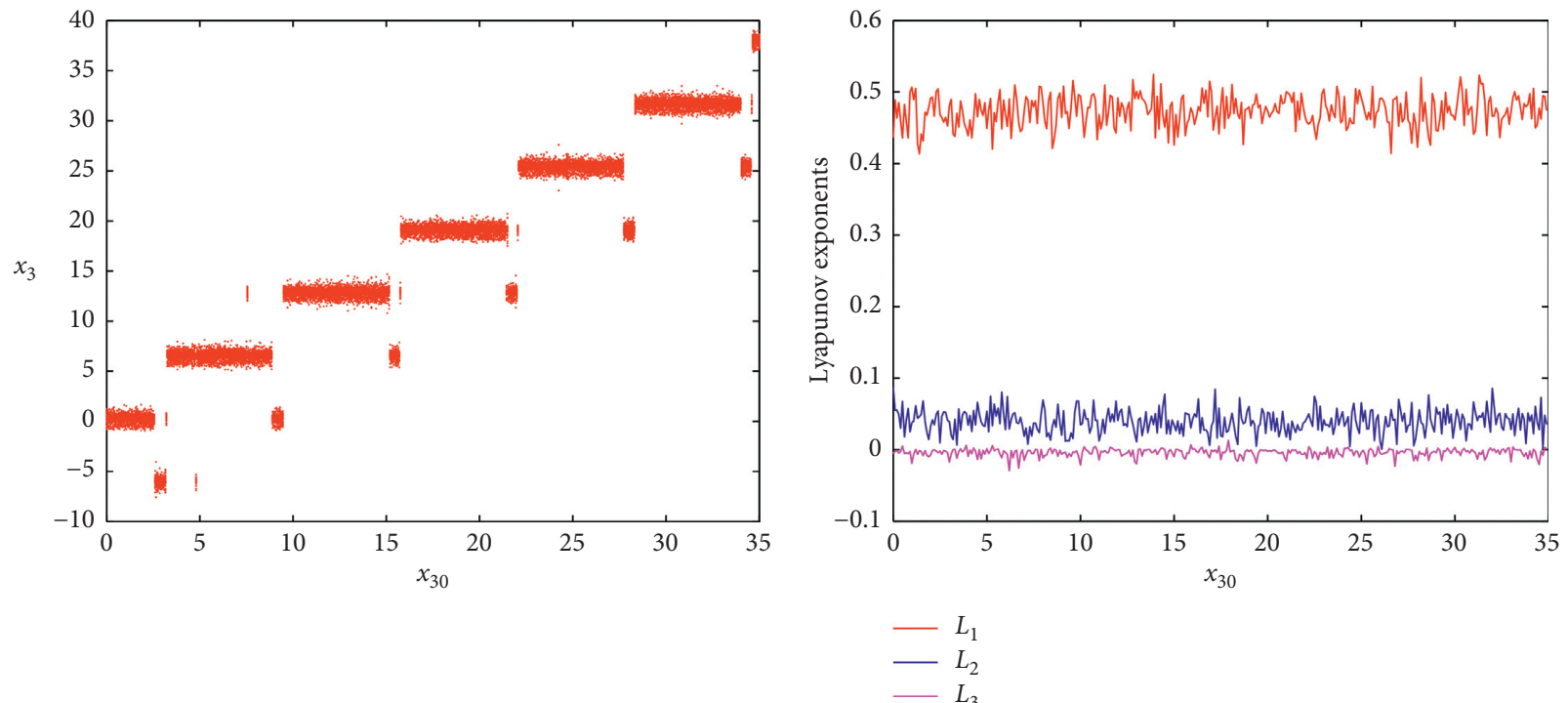

(a)

(b)

FIGURE 7: Bifurcation diagram and Lyapunov exponent of system (2) with $a=4, b=c=1, k=12$, and $x_{30} \in[0,35]$, where $L_{1}>L_{2}>L_{3}>L_{4}$ are Lyapunov exponents of system (2) and $L_{4}$ is always less than -4 . 


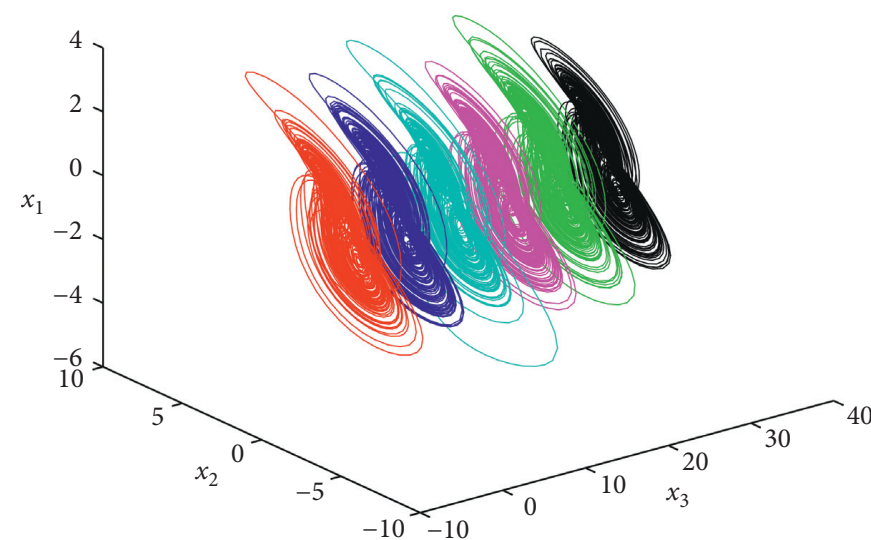

(a)

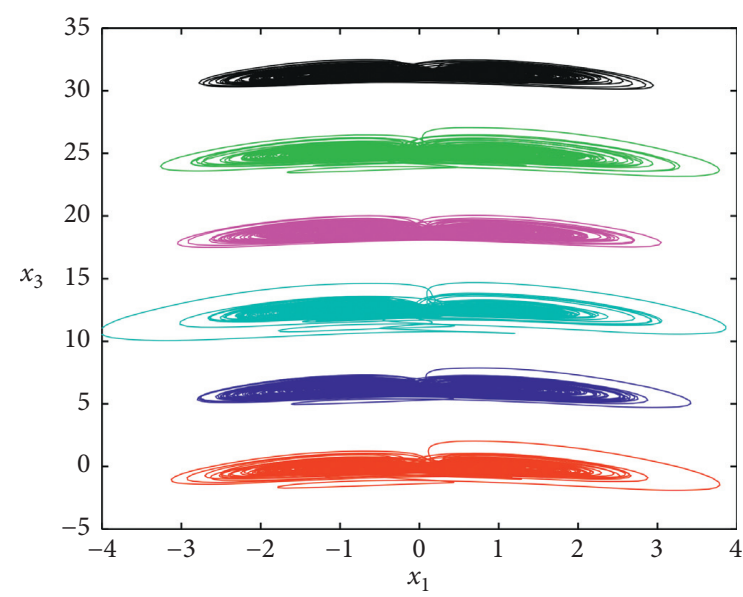

(b)

Figure 8: Coexisting six hidden strange attractors of system (2) with $a=4, b=c=1$, and $k=12$ and initial values ( $1,1,2 j \pi, 1)$, $j=0,1,2,3,4,5$.

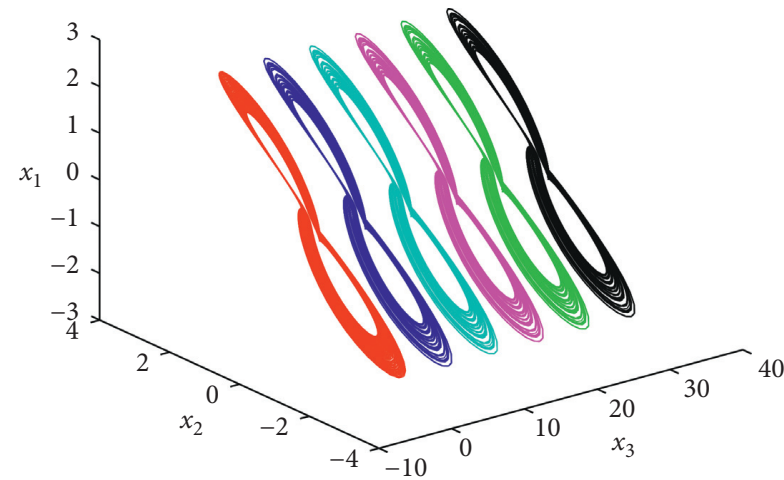

(a)

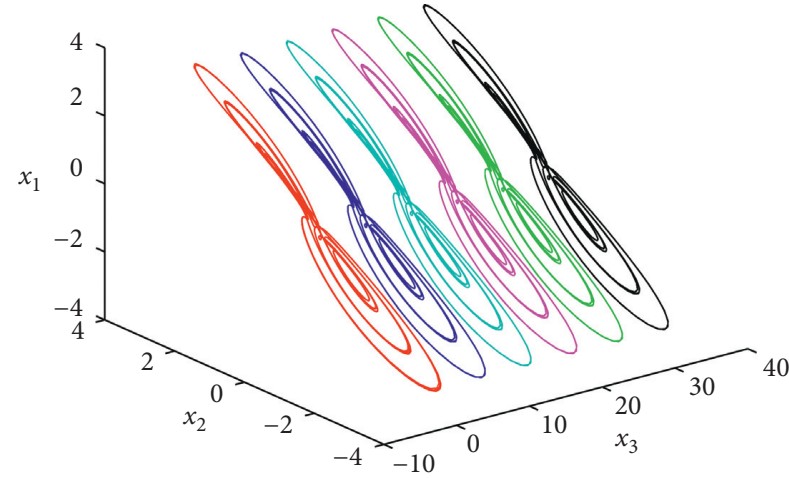

(b)

FiguRE 9: Coexisting six hidden attractors of system (2) from initial values $(1,1,2 j \pi, 1), j=0,1,2,3,4,5$. (a) Quasiperiodic attractors for $a=12, b=c=1$, and $k=12$. (b) Periodic attractors for $a=20, b=c=1$, and $k=12$.

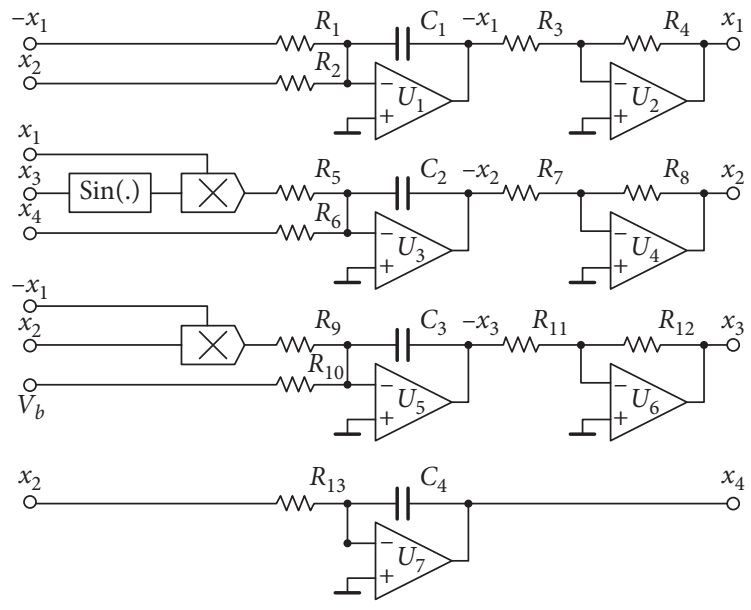

Figure 10: Analog circuit of system (2).

$$
\left\{\begin{array}{l}
\dot{x}_{1}=a\left(x_{2}-x_{1}\right), \\
\dot{x}_{2}=k x_{1} \sin \left(x_{3}\right)+x_{4}, \\
\dot{x}_{3}=b-x_{1} x_{2}, \\
\dot{x}_{4}=-c x_{2}
\end{array}\right.
$$

and the slave system is given by

$$
\left\{\begin{array}{l}
\dot{y}_{1}=a\left(y_{2}-y_{1}\right)+u_{1}, \\
\dot{y}_{2}=k y_{1} \sin \left(y_{3}\right)+y_{4}+u_{2}, \\
\dot{y}_{3}=b-y_{1} y_{2}+u_{3}, \\
\dot{y}_{4}=-c y_{2}+u_{4},
\end{array}\right.
$$

where $u=\left(u_{1}, u_{2}, u_{3}, u_{4}\right)^{T}$ is the adaptive control law to be designed. The parameters of the slave system are all unknown and the design goal is to find the control feedback law 


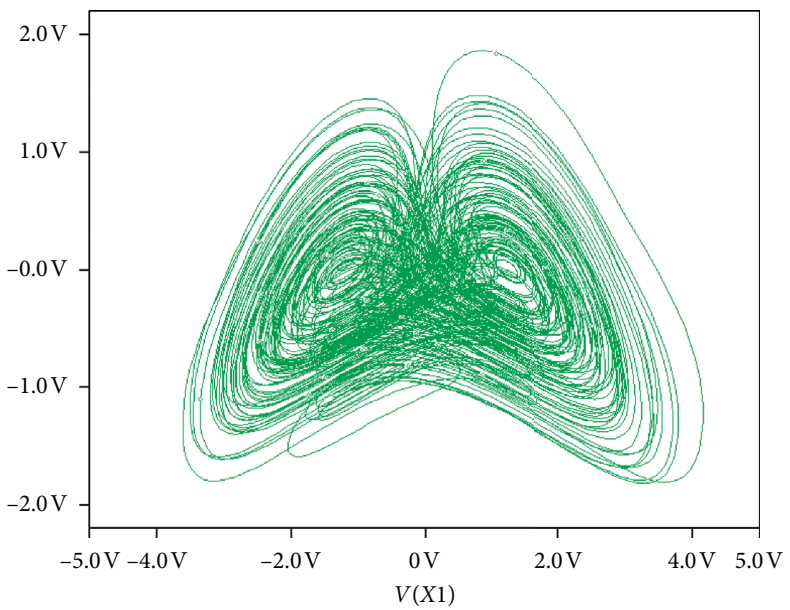

\& $V(X 3)$

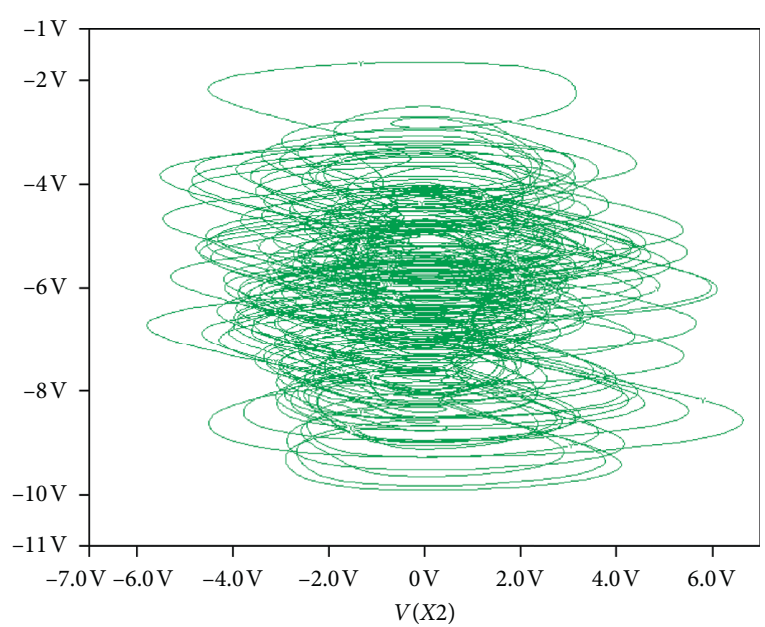

$\curlyvee V(X 4)$

(a)

(b)

FiguRE 11: A chaotic attractor of system (2) obtained from the analog circuit for $R_{a}=25 \mathrm{k} \Omega, R_{5}=8.3333 \mathrm{k} \Omega, R_{13}=100 \mathrm{k} \Omega$, and $V_{b}=1 \mathrm{~V}$ : (a) $x_{1}-x_{3}$; (b) $x_{2}-x_{4}$.

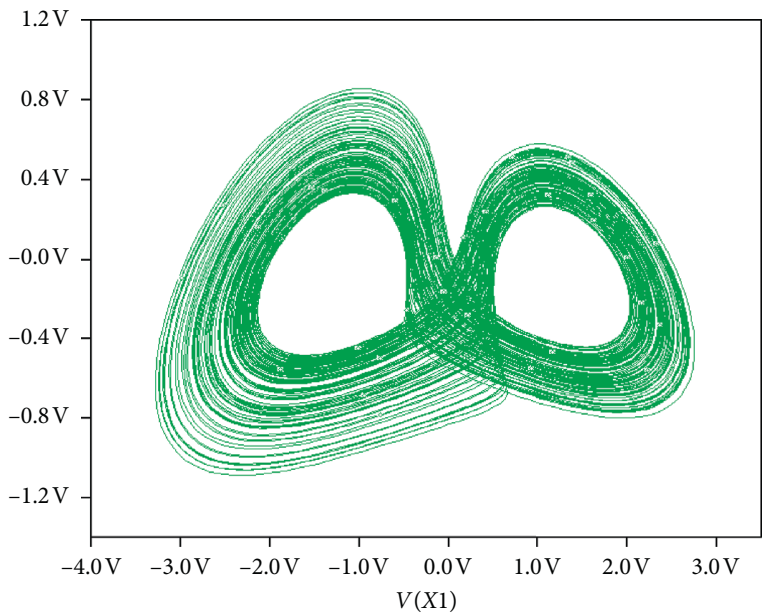

$\Leftrightarrow V(X 3)$

(a)

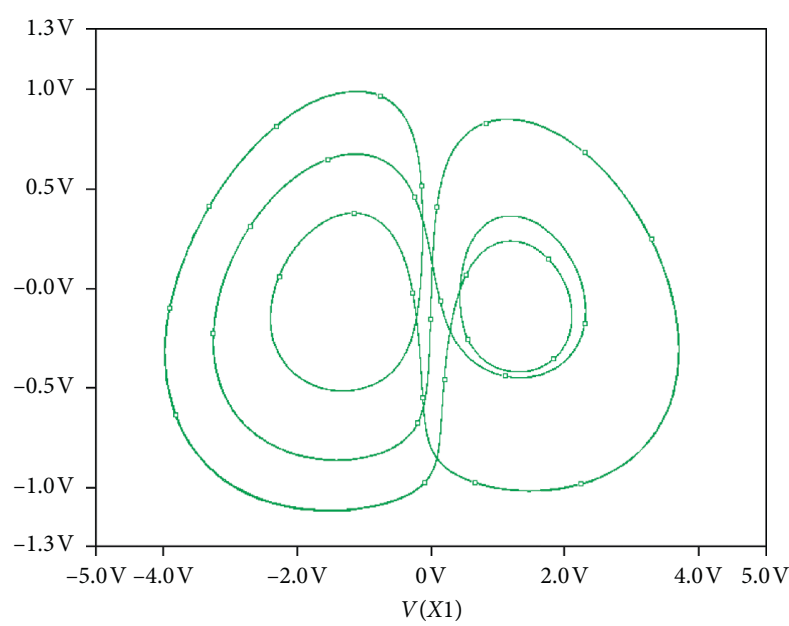

$V(X 3)$

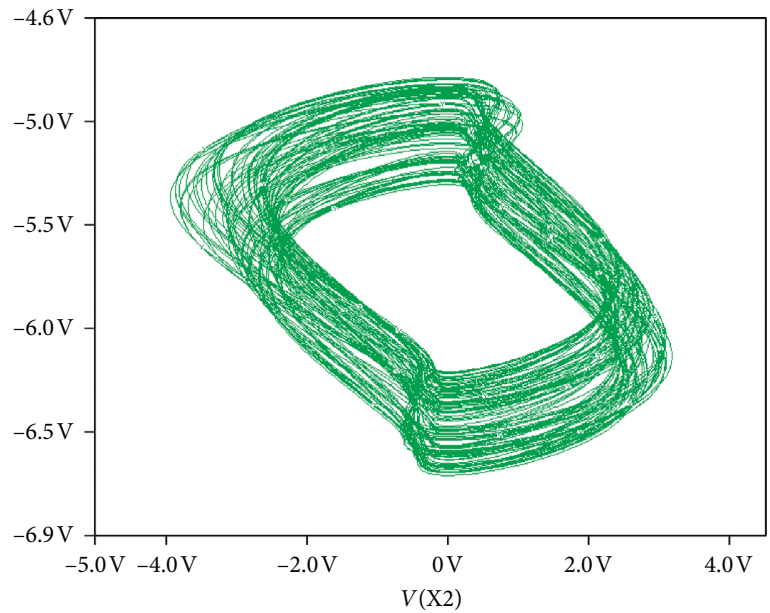

$Y V(X 4)$

(b)

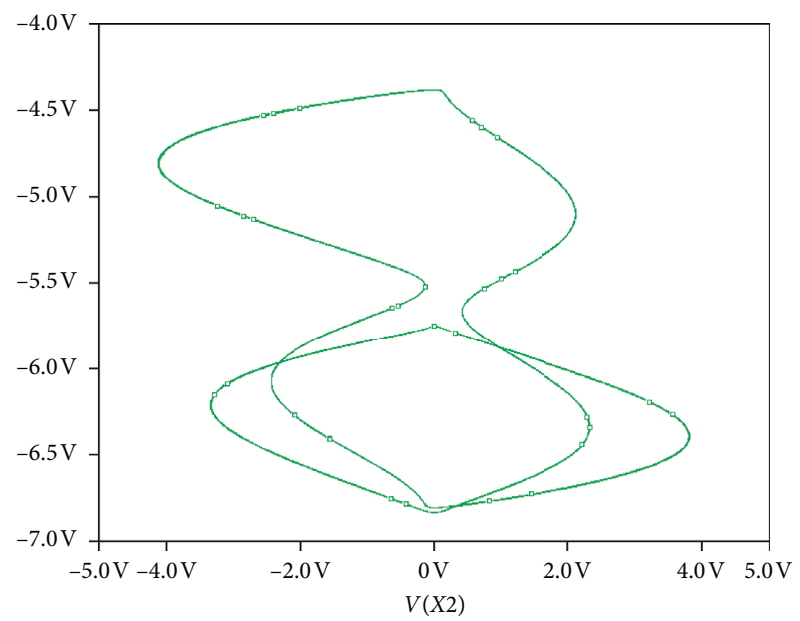

$V(X 4)$

(c)

(d)

Figure 12: Quasiperiodic motion observed for $R_{a}=10 k \Omega, R_{5}=8.3333 k \Omega, R_{13}=100 k \Omega$, and $V_{b}=1 \mathrm{~V}$ : (a) $x_{1}-x_{3}$; (b) $x_{2}-x_{4}$. Periodic motion observed for $R_{a}=2.65 \mathrm{k} \Omega, R_{5}=8.3333 \mathrm{k} \Omega, R_{13}=100 \mathrm{k} \Omega$, and $V_{b}=1 \mathrm{~V}$ : (c) $x_{1}-x_{3}$; (d) $x_{2}-x_{4}$. 


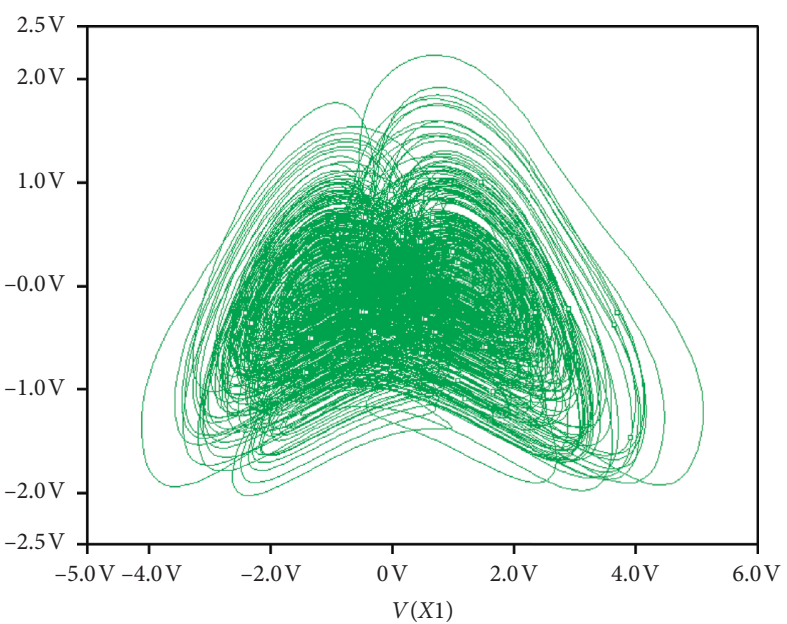

$\square V(X 3)$

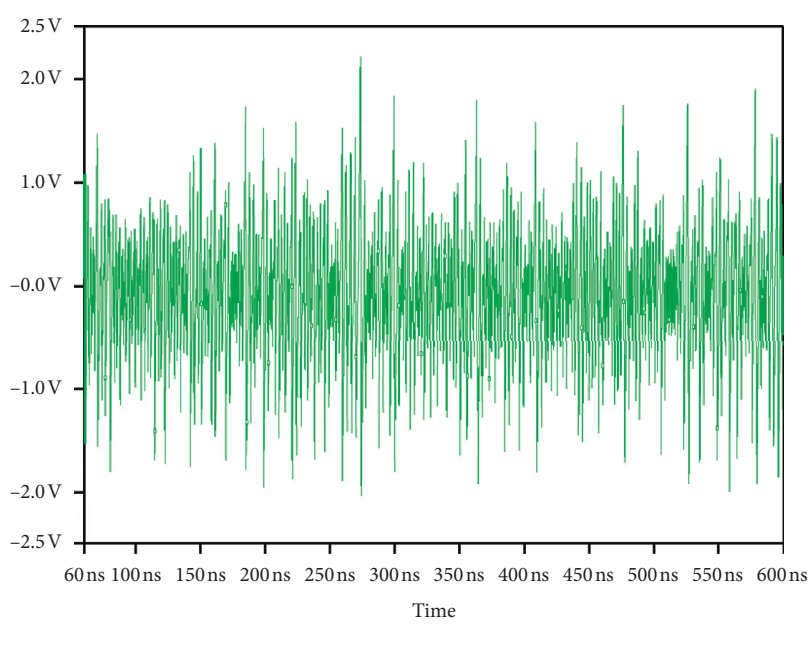

(b)

FiguRe 13: Chaotic behavior of system (2) observed for $R_{a}=25 k \Omega, R_{5}=8.3333 k \Omega, R_{13}=8.333 k \Omega$, and $V_{b}=0.8 \mathrm{~V}$ : (a) $x_{1}-x_{3}$; (b) $x_{2}-x_{4}$.

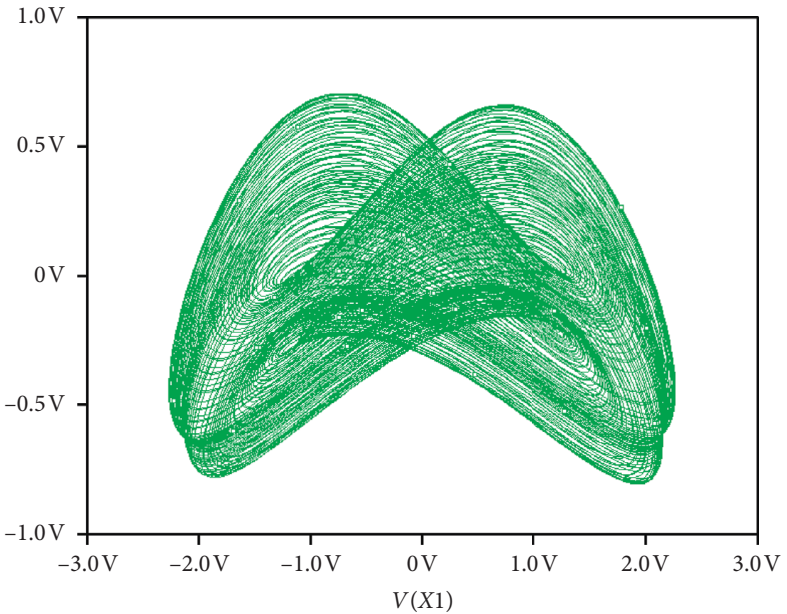

$\square V(X 3)$

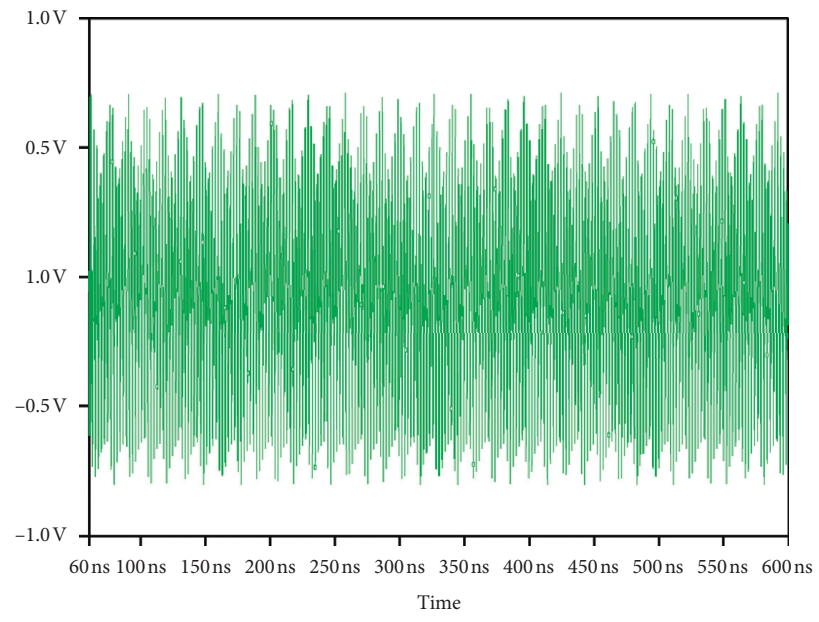

ㅁ $V(X 3)$

Figure 14: Periodic orbit observed from the analog circuit of the novel system for $R_{a}=25 k \Omega, R_{5}=8.33334 k \Omega, R_{13}=16.6667 k \Omega$, and $V_{b}=0.8 \mathrm{~V}$ : (a) $x_{1}-x_{3}$; (b) $x_{2}-x_{4}$.

$u$ to eliminate the error between the master system and the slave system. The synchronization error is defined as follows:

$$
\left\{\begin{array}{l}
e_{1}=y_{1}-x_{1}, \\
e_{2}=y_{2}-x_{2}, \\
e_{3}=y_{3}-x_{3}, \\
e_{4}=y_{4}-x_{4} .
\end{array}\right.
$$

The corresponding error system is written as

$$
\left\{\begin{array}{l}
\dot{e}_{1}=a\left(e_{2}-e_{1}\right)+u_{1}, \\
\dot{e}_{2}=k\left(y_{1} \sin \left(y_{3}\right)-x_{1} \sin \left(x_{3}\right)\right)+e_{4}+u_{2}, \\
\dot{e}_{3}=x_{1} x_{2}-y_{1} y_{2}+u_{3}, \\
\dot{e}_{4}=-c e_{2}+u_{4} .
\end{array}\right.
$$

Give the adaptive feedback control laws as follows:

$\left\{\begin{array}{l}u_{1}=-\widehat{a}(t)\left(e_{2}-e_{1}\right)-g_{1} e_{1}, \\ u_{2}=-\widehat{k}(t)\left(y_{1} \sin \left(y_{3}\right)-x_{1} \sin \left(x_{3}\right)\right)-e_{4}-g_{2} e_{2}, \\ u_{3}=-x_{1} x_{2}+y_{1} y_{2}-g_{3} e_{3}, \\ u_{4}=\widehat{c}(t) e_{2}-g_{4} e_{4},\end{array}\right.$ 


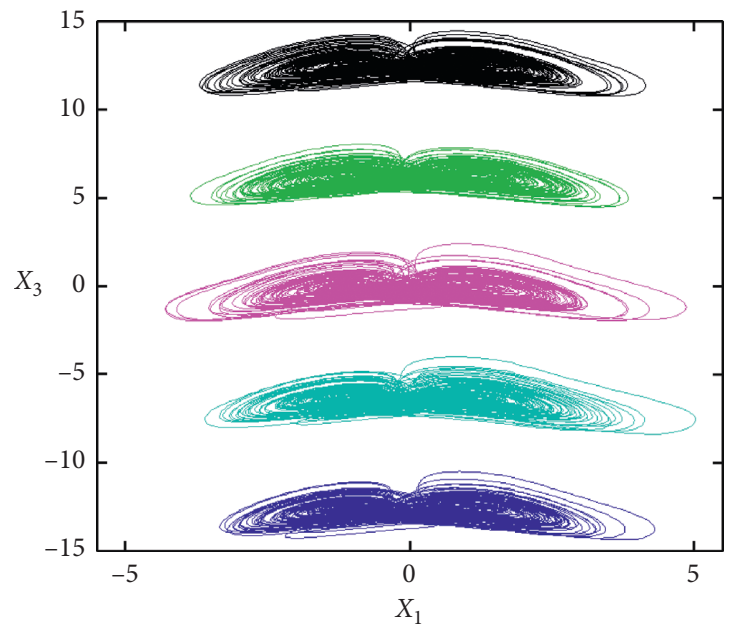

(a)

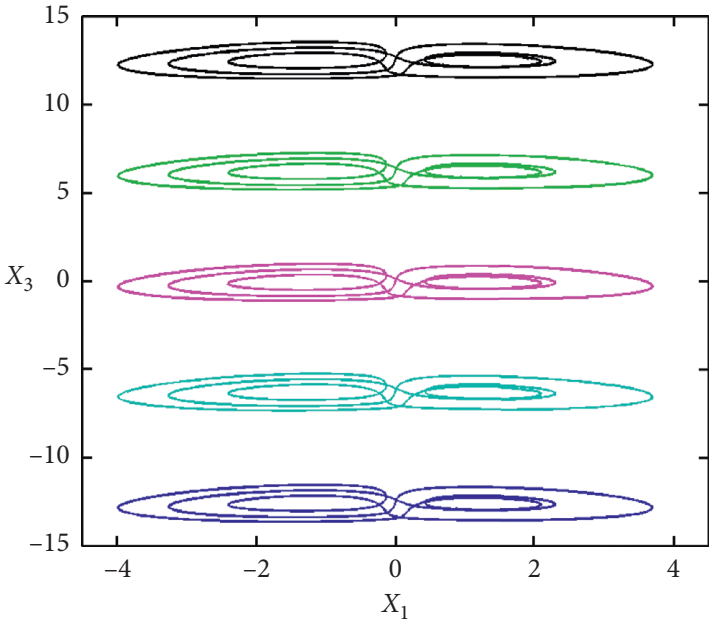

(b)

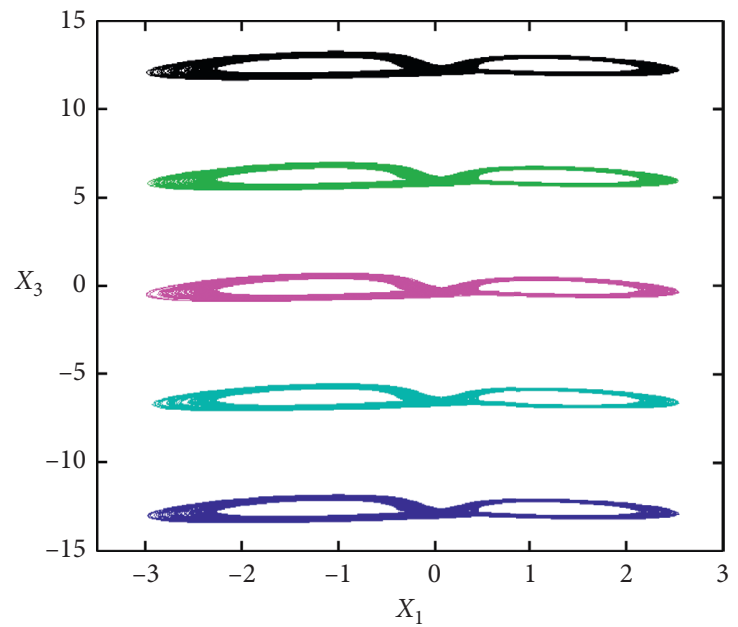

(c)

FIGURE 15: Projections on $x_{1}-x_{3}$ plane of coexisting attractors obtained from the analog circuit of system (2) for initial voltage of capacitor $C_{3}$ fixed as $(1,1,-6 \pi),(1,1,-4 \pi),(1,1,-2 \pi),(1,1,0),(1,1,2 \pi)$, and $(1,1,4 \pi)$ : (a) coexisting five chaotic attractors; (b) coexisting five periodic attractors; (c) coexisting five quasiperiodic attractors.

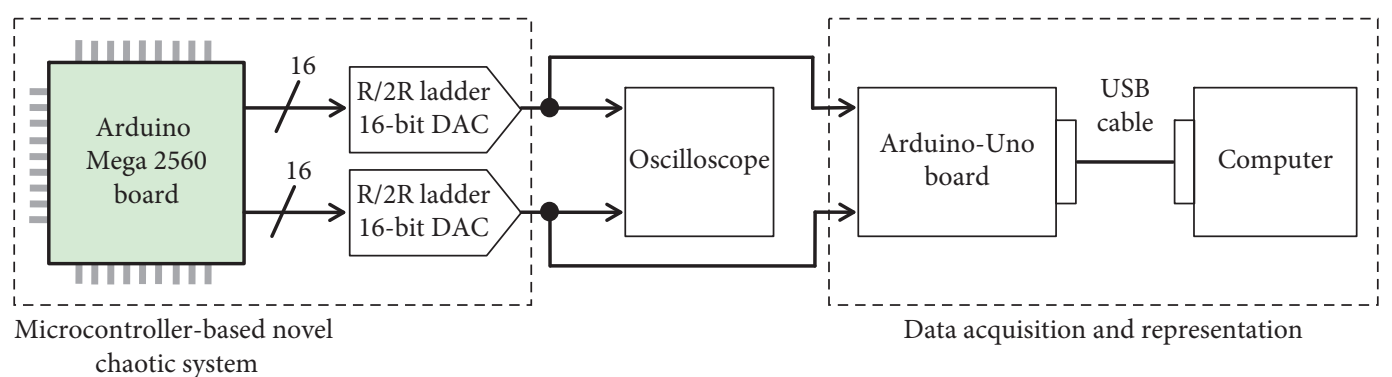

FIGURE 16: Scheme of the experiment on microcontroller-based implementation of system (2). 


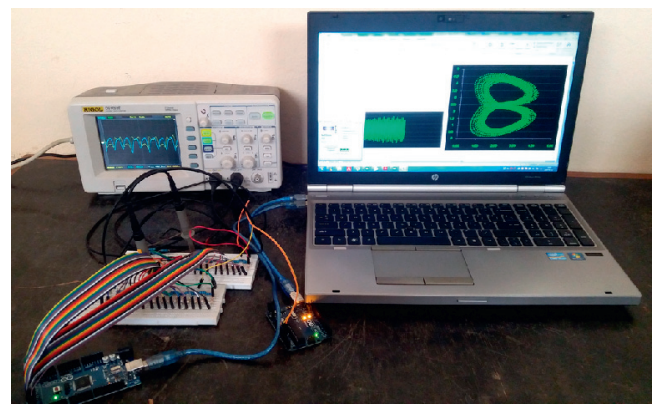

Figure 17: Practical setup for the experiment on the microcontroller-based on the implementation of system (2).

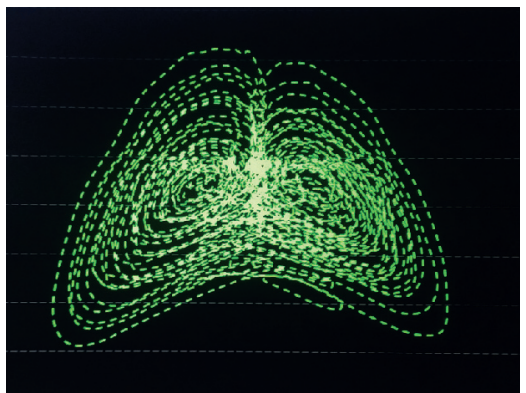

(a)

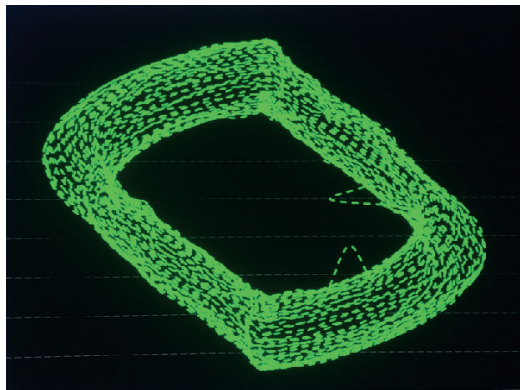

(d)

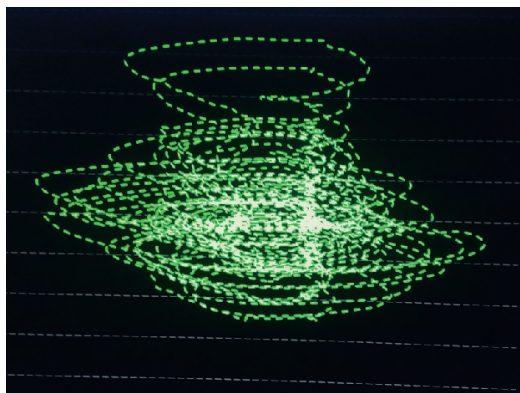

(b)

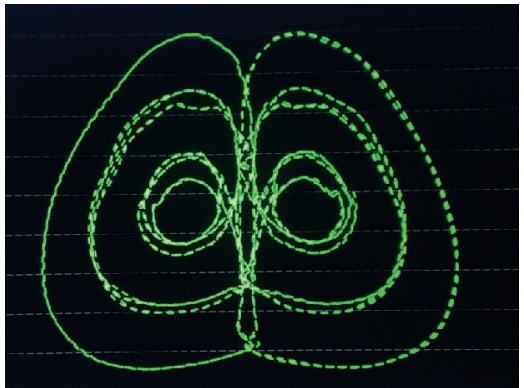

(e)

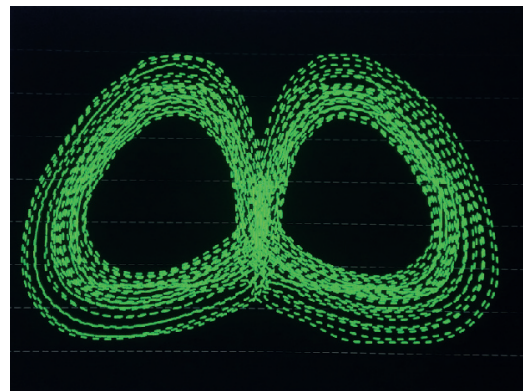

(c)

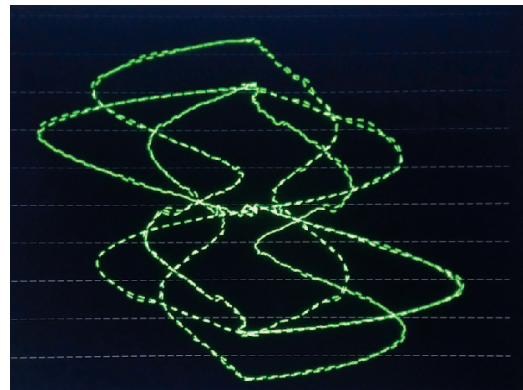

(f)

FiguRE 18: Experimental phase portraits from the microcontroller-based implementation of system (2): (a, b) projections on $x_{1}-x_{3}$ and $x_{2}-x_{4}$ planes for chaotic attractor; (c, d) projections on $x_{1}-x_{3}$ and $x_{2}-x_{4}$ planes for quasiperiodic attractor; (e, $\left.\mathrm{f}\right)$ projections on $x_{1}-x_{3}$ and $x_{2}-x_{4}$ planes for periodic attractor.

where $g_{1}, g_{2}, g_{3}$, and $g_{4}$ are positive gain constants. By substituting the control laws $u_{1}, u_{2}, u_{3}$, and $u_{4}$ into system (7), we get

$$
\left\{\begin{array}{l}
\dot{e}_{1}=(a-\widehat{a}(t))\left(e_{2}-e_{1}\right)-g_{1} e_{1}, \\
\dot{e}_{2}=(k-\widehat{k}(t))\left(y_{1} \sin \left(y_{3}\right)-x_{1} \sin \left(x_{3}\right)\right)-g_{2} e_{2}, \\
\dot{e}_{3}=-g_{3} e_{3}, \\
\dot{e}_{4}=-(c-\widehat{c}(t)) e_{2}-g_{4} e_{4} .
\end{array}\right.
$$

The errors in system (9) can be simplified by taking the parameter estimation errors as

$$
\left\{\begin{array}{l}
e_{a}(t)=a-\widehat{a}(t), \\
e_{b}(t)=b-\widehat{b}(t), \\
e_{c}(t)=c-\widehat{c}(t), \\
e_{k}(t)=k-\widehat{k}(t) .
\end{array}\right.
$$

It follows that

$$
\left\{\begin{array}{l}
\dot{e}_{a}=-\widehat{a}(t), \\
\dot{e}_{b}=-\widehat{b}(t), \\
\dot{e}_{c}=-\widehat{c}(t) \\
\dot{e}_{k}=-\widehat{k}(t) .
\end{array}\right.
$$

Thus, system (9) can be rewritten as

$$
\left\{\begin{array}{l}
\dot{e}_{1}=e_{a}\left(e_{2}-e_{1}\right)-k_{1} e_{1}, \\
\dot{e}_{2}=e_{k}\left(y_{1} \sin \left(y_{3}\right)-x_{1} \sin \left(x_{3}\right)\right)-k_{2} e_{2}, \\
\dot{e}_{3}=-k_{3} e_{3}, \\
\dot{e}_{4}=-e_{c} e_{2}-k_{4} e_{4} .
\end{array}\right.
$$

Next we will establish the synchronization conditions by using the Lyapunov function. Consider the Lyapunov function 


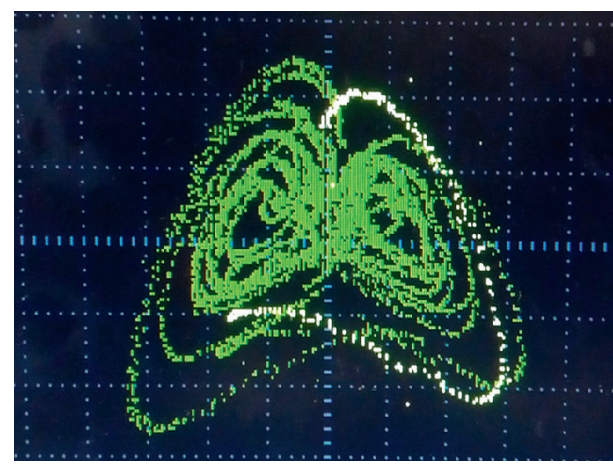

(a)

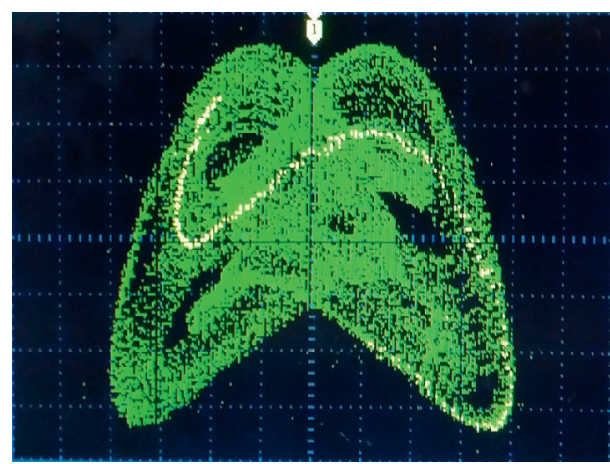

(c)

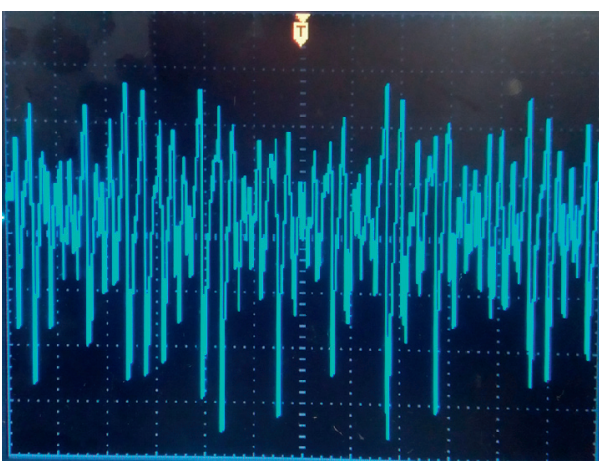

(b)

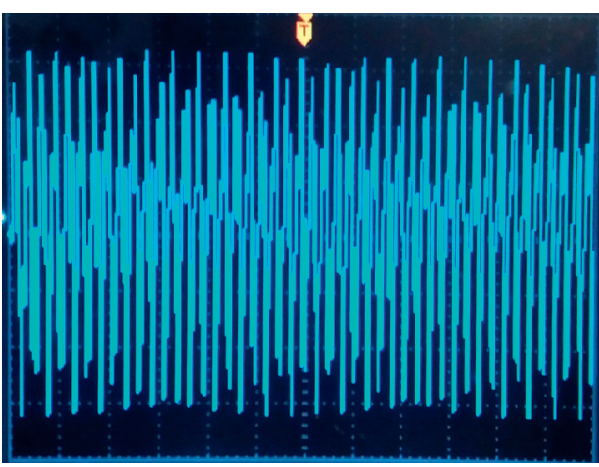

(d)

FIGURE 19: Chaotic and quasiperiodic attractors from the microcontroller-based implementation of system (2).

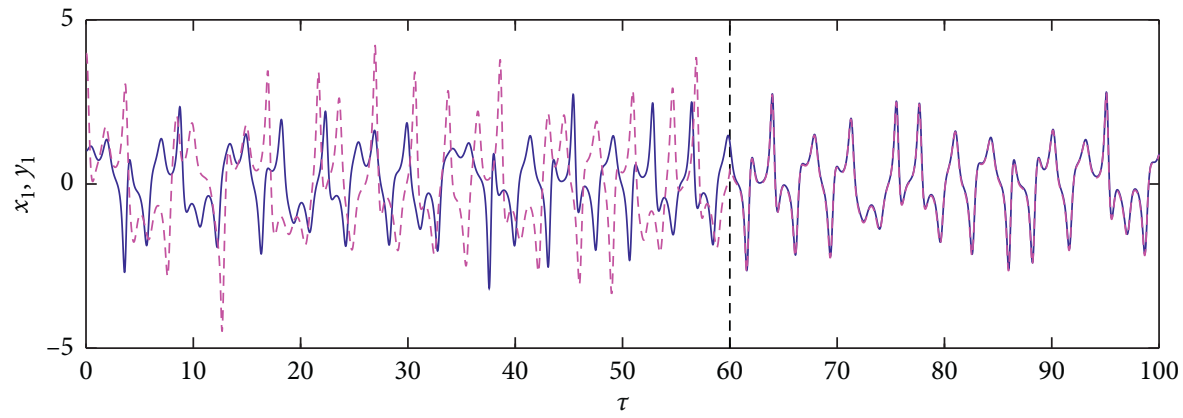

(a)

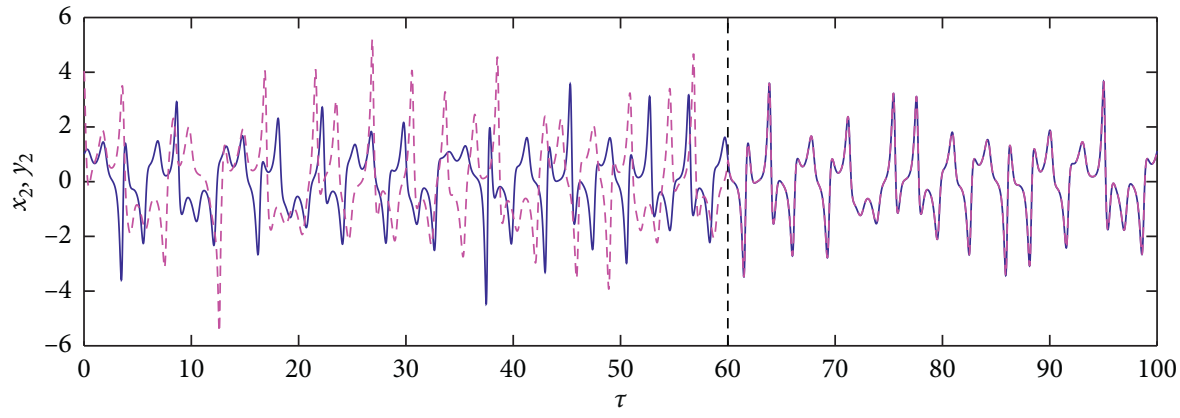

(b)

FIgURe 20: Continued. 


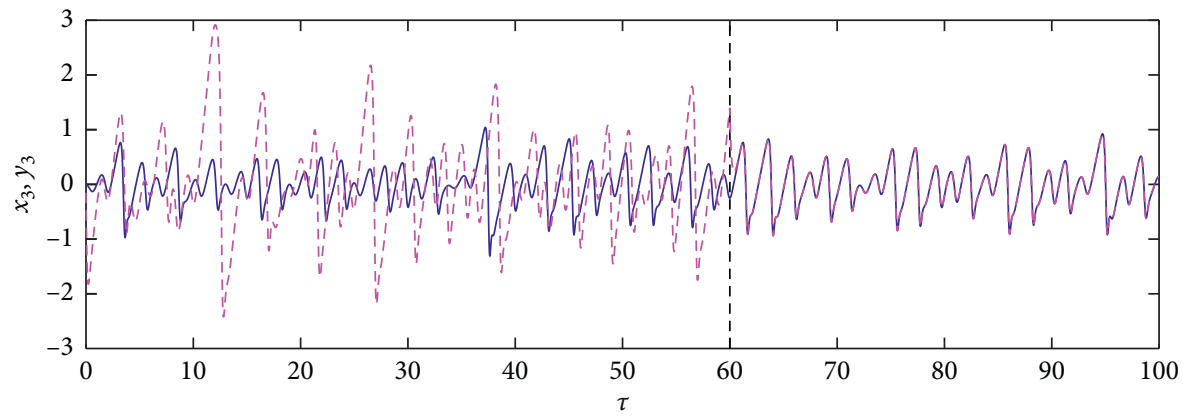

(c)

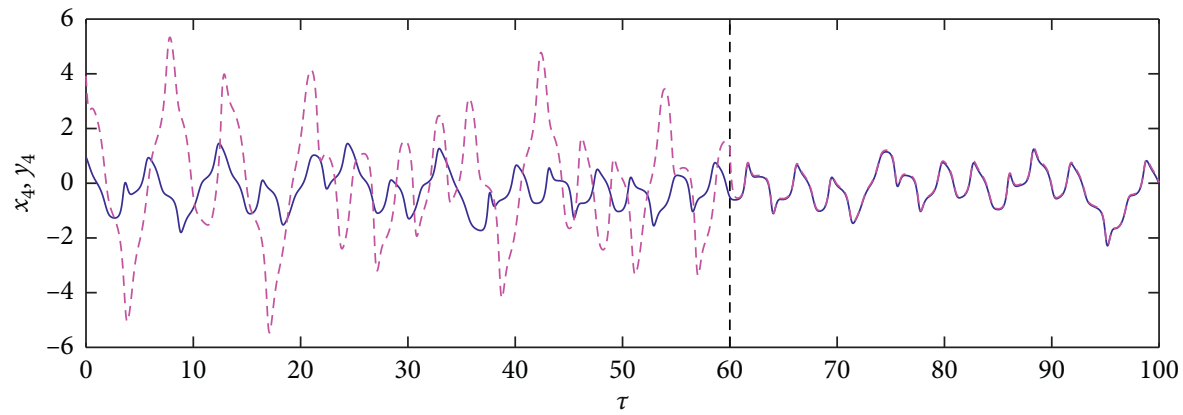

(d)

FIGURE 20: Time series $x_{1}(\tau), x_{2}(\tau), x_{3}(\tau)$, and $x_{4}(\tau)$ of master and slave systems showing the result of the adaptive law activated at $\tau=60$.

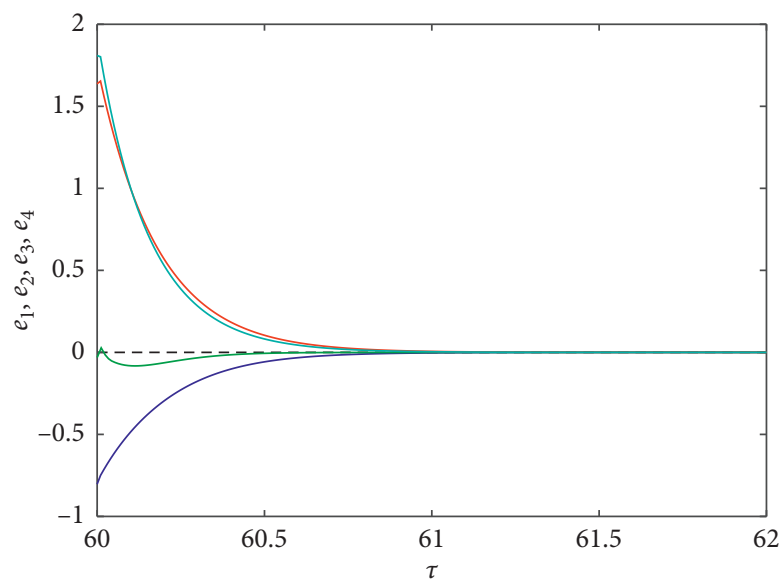

$-e_{1}$

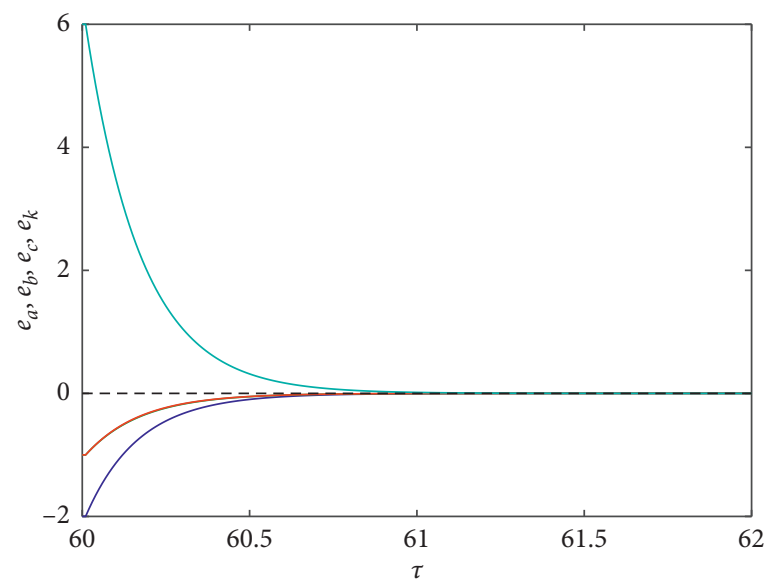

$-e_{a}$

(a)

(b)

Figure 21: Time evolution of the synchronization errors $e_{1}, e_{2}, e_{3}$, and $e_{4}$ and the parameter estimation errors $e_{a}, e_{b}, e_{c}$, and $e_{k}$ directly after the activation of the adaptive control law.

$$
V=\frac{1}{2}\left(e_{1}^{2}+e_{2}^{2}+e_{3}^{2}+e_{4}^{2}+e_{a}^{2}+e_{b}^{2}+e_{c}^{2}+e_{k}^{2}\right) .
$$

Differentiating $V$ along the trajectories, we have

$$
\begin{aligned}
\dot{V}= & -g_{1} e_{1}^{2}-g_{2} e_{2}^{2}-g_{3} e_{3}^{2}-g_{4} e_{4}^{2}+e_{a}\left[e_{1}\left(e_{2}-e_{1}\right)-\dot{\hat{a}}\right] \\
& -e_{b} \dot{\hat{b}}-e_{c}\left(e_{2} e_{4}+\dot{\hat{c}}\right)+e_{k}\left[e_{2}\left(y_{1} \sin \left(y_{3}\right)-x_{1} \sin \left(x_{3}\right)\right)-\dot{\hat{k}}\right] .
\end{aligned}
$$


Here, the parameter update laws are

$$
\left\{\begin{array}{l}
\dot{\hat{a}}=e_{1}\left(e_{2}-e_{1}\right)+g_{5} e_{a}, \\
\dot{\hat{b}}=g_{6} e_{b}, \\
\dot{\hat{c}}=-e_{2} e_{4}+g_{7} e_{c}, \\
\dot{\hat{k}}=e_{2}\left(y_{1} \sin \left(y_{3}\right)-x_{1} \sin \left(x_{3}\right)\right)+g_{8} e_{k},
\end{array}\right.
$$

with positive gain constants $g_{5}, g_{6}, g_{7}$, and $g_{8}$. By substituting the parameter update law (15) into (14), we have $\dot{V}=-g_{1} e_{1}^{2}-g_{2} e_{2}^{2}-g_{3} e_{3}^{2}-g_{4} e_{4}^{2}-g_{5} e_{a}^{2}-g_{6} e_{b}^{2}-g_{7} e_{c}^{2}-g_{8} e_{k}^{2}$.

Based on the Lyapunov stability theory, we can derive that the synchronization errors $e_{1}, e_{2}, e_{3}$, and $e_{4}$ and the parameter estimation errors $e_{a}, e_{b}, e_{c}$, and $e_{k}$ globally and exponentially converge to zero with time increasing. Thus, we obtained the following theorem.

Theorem 1. The systems in (4) and (5) with unknown parameters are globally and exponentially synchronized for all initial conditions by the adaptive feedback control law (8) and the parameter update law (15), where $g_{i},(i=1,2, \ldots, 8)$ are positive constants. The parameter estimation errors $e_{a}, e_{b}, e_{c}$, and $e_{k}$ globally and exponentially converge to zero with time.

Next, we will verify the effectiveness of Theorem 1 via numerical simulation. Suppose the parameters $a=6, b=1$, $c=1$, and $k=12$ of system (4) and system (5). The systems are integrated via the fourth order Runge-Kutta method from 0 to 100 with a time step $\Delta t=0.001$. The control law (8) is exactly applied at $\tau=60$. Initial values are randomly taken as $(1,1,0,1)$ for system $(4)$ and $(4,4,0,4)$ for system $(5)$. The initial values of parameters are selected randomly as $\widehat{a}(0)=8, \widehat{b}(0)=2, \widehat{c}(0)=2$, and $\widehat{k}(0)=6$. The time series of signals from the master and slave systems are shown in Figure 19. The synchronization errors and the parameters estimations are also presented in Figure 20. From the numerical simulations, one can see that the adaptive synchronization between master system (4) and slave system (5) is successfully achieved and the error signals asymptotically approach zero. The time evolution of the synchronization errors and the parameter estimation errors is presented in Figure 21. As one can see, after the activation of the control law, the errors all converge to zero, showing that the parameters meet the instructed value and the master and slave systems exhibit the same dynamic behavior.

\section{Conclusions}

This paper focuses on the dynamic analysis, circuit, and microcontroller realization and synchronization control of a new chaotic system with infinite many coexisting hidden attractors. The system generated from an augmented Sprott B system has no equilibrium. Its distinct feature is that it can generate any number of hidden attractors for selecting different initial values. Also, it exhibits chaotic, periodic, and quasiperiodic motion with the variation of parameters. The circuit and microcontroller realization is given for illustrating the dynamic behaviors of the system. Moreover, the adaptive synchronization conditions of the system are theoretically and numerically established.

\section{Data Availability}

The data used to support the findings of this study are available from the corresponding author upon request.

\section{Conflicts of Interest}

The authors declare that there are no conflicts of interest regarding the publication of this paper.

\section{Acknowledgments}

This work was supported by the National Natural Science Foundation of China under Grant nos. 61961019 and 61803222 and the Key Research and Development Program of Jiangxi Province of China under Grant no. 20181BBE50017.

\section{References}

[1] E. N. Lorenz, "Deterministic nonperiodic flow," Journal of the Atmospheric Sciences, vol. 20, no. 2, pp. 130-141, 1963.

[2] O. E. Rössler, "An equation for continuous chaos," Physics Letters A, vol. 57, no. 5, pp. 397-398, 1976.

[3] G. Chen and T. Ueta, "Yet another chaotic attractor," International Journal of Bifurcation and Chaos, vol. 9, no. 7, pp. 1465-1466, 1999.

[4] C. Li, W. Joo-Chen Thio, H. H. C. Iu, and L. Tian, "A memristive chaotic oscillator with increasing amplitude and frequency," IEEE Access, vol. 6, pp. 12945-12950, 2018.

[5] Q. Lai, P. D. Kamdem Kuate, F. Liu, and H. H. C. Iu, "An extremely simple chaotic system with infinitely many coexisting attractors," IEEE Transactions on Circuits and Systems II: Express Briefs, vol. 68, 2019.

[6] C.-L. Li, H.-M. Li, W. Li et al., "Dynamics, implementation and stability of a chaotic system with coexistence of hyperbolic and non-hyperbolic equilibria," AEU - International Journal of Electronics and Communications, vol. 84, pp. 199-205, 2018.

[7] C. Li, K. Qian, S. He, H. Li, and W. Feng, "Dynamics and optimization control of a robust chaotic map," IEEE Access, vol. 7, pp. 160072-160081, 2019.

[8] J. C. Sprott, "Some simple chaotic flows," Physical Review E, vol. 50, no. 2, pp. 647-650, 1994.

[9] S. Dadras and H. R. Momeni, "A novel three-dimensional autonomous chaotic system generating two, three and fourscroll attractors," Physics Letters A, vol. 373, no. 40, pp. 3637-3642, 2009.

[10] N. B. Slimane, K. Bouallegue, and M. Machhout, "Designing a multi-scroll chaotic system by operating Logistic map with fractal process," Nonlinear Dynamics, vol. 88, no. 3, pp. 1655-1675, 2017.

[11] C. Zhang, S. Yu, Q. He, and J. Ruan, "On constructing complex grid multiwing chaotic system by switching control and mirror symmetry conversion," International Journal of Bifurcation and Chaos, vol. 23, no. 7, Article ID 1350115, 2013.

[12] Q. Lai, A. Akgul, C. B. Li, G. Xu, and U. Cavusoglu, "A new chaotic system with multiple attractors: dynamic analysis, 
circuit realization and S-Box design," Entropy, vol. 20, no. 1, p. 12, 2017.

[13] Q. Lai, B. Norouzi, and F. Liu, "Dynamic analysis, circuit realization, control design and image encryption application of an extended Lü system with coexisting attractors," Chaos, Solitons \& Fractals, vol. 114, pp. 230-245, 2018.

[14] H. Wu, H. Bao, Q. Xu, and M. Chen, "Abundant coexisting multiple attractors' behaviors in three-dimensional sine chaotic system," Complexity, vol. 2019, Article ID 3687635, 11 pages, 2019.

[15] C. P. Silva, "Shil'nikov's theorem-a tutorial," IEEE Transactions on Circuits and Systems I: Fundamental Theory and Applications, vol. 40, no. 10, pp. 675-682, 1993.

[16] S. Jafari and J. C. Sprott, "Simple chaotic flows with a line equilibrium," Chaos, Solitons \& Fractals, vol. 57, no. 4, pp. 79-84, 2013.

[17] S. Jafari, J. C. Sprott, and S. M. R. Hashemi Golpayegani, "Elementary quadratic chaotic flows with no equilibria," Physics Letters A, vol. 377, no. 9, pp. 699-702, 2013.

[18] T. Gotthans and J. Petrzela, "New class of chaotic systems with circular equilibrium," Nonlinear Dynamics, vol. 81, no. 33, pp. 1143-1149, 2015.

[19] J. C. Sprott, S. Jafari, V.-T. Pham, and Z. S. Hosseini, "A chaotic system with a single unstable node," Physics Letters A, vol. 379, no. 36, pp. 2030-2036, 2015.

[20] C. Li and J. C. Sprott, "Multistability in the Lorenz system: a broken butterfly," International Journal of Bifurcation and Chaos, vol. 24, no. 10, Article ID 1450131, 2014.

[21] C. Li, J. C. Sprott, and H. Xing, "Constructing chaotic systems with conditional symmetry," Nonlinear Dynamics, vol. 87, no. 2, pp. 1351-1358, 2017.

[22] J. Kengne, Z. T. Njitacke, and H. B. Fotsin, "Dynamical analysis of a simple autonomous jerk system with multiple attractors," Nonlinear Dynamics, vol. 83, no. 1-2, pp. 751-765, 2016.

[23] Q. Lai and S. Chen, "Generating multiple chaotic attractors from Sprott B system," International Journal of Bifurcation and Chaos, vol. 26, no. 11, Article ID 1650177, 2016.

[24] Q. Lai, C. Chen, X.-W. Zhao, J. Kengne, and C. Volos, "Constructing chaotic system with multiple coexisting attractors," IEEE Access, vol. 7, no. 1, pp. 24051-24056, 2019.

[25] C. Li, W. Joo-Chen Thio, J. C. Sprott, H. H.-C. Iu, and Y. Xu, "Constructing infinitely many attractors in a programmable chaotic circuit," IEEE Access, vol. 6, pp. 29003-29012, 2018.

[26] B. C. Bao, P. Y. Wu, H. Bao, H. G. Wu, X. Zhang, and M. Chen, "Symmetric periodic bursting behavior and bifurcation mechanism in a third-order memristive diode bridgebased oscillator," Chaos, Solitons \& Fractals, vol. 109, pp. 146-153, 2018.

[27] B. Bao, L. Xu, Z. Wu, M. Chen, and H. Wu, "Coexistence of multiple bifurcation modes in memristive diode-bridge-based canonical Chua's circuit," International Journal of Electronics, vol. 105, no. 7, pp. 1159-1169, 2018.

[28] C. Li, Y. Zhou, Y. Yang et al., "Complicated dynamics in a memristor-based RLC circuit," The European Physical Journal Special Topics, vol. 228, no. 10, pp. 1925-1941, 2019.

[29] C.-L. Li, Z.-Y. Li, W. Feng, Y.-N. Tong, J.-R. Du, and D.-Q. Wei, "Dynamical behavior and image encryption application of a memristor-based circuit system," AEU-International Journal of Electronics and Communications, vol. 110, p. 152861, 2019.

[30] Z. Wei, F. Parastesh, H. Azarnoush et al., "Nonstationary chimeras in a neuronal network," EPL (Europhysics Letters), vol. 123, no. 4, p. 48003, 2018.
[31] D. Dudkowski, S. Jafari, T. Kapitaniak, N. V. Kuznetsov, G. A. Leonov, and A. Prasad, "Hidden attractors in dynamical systems," Physics Reports, vol. 637, pp. 1-50, 2016.

[32] M. A. Kiseleva, N. V. Kuznetsov, and G. A. Leonov, "Hidden attractors in electromechanical systems with and without equilibria," IFAC-PapersOnLine, vol. 49, no. 14, pp. 51-55, 2016.

[33] G. A. Leonov, N. V. Kuznetsov, M. A. Kiseleva, E. P. Solovyeva, and A. M. Zaretskiy, "Hidden oscillations in mathematical model of drilling system actuated by induction motor with a wound rotor," Nonlinear Dynamics, vol. 77, no. 1-2, pp. 277-288, 2014.

[34] G. A. Leonov and N. V. Kuznetsov, "On differences and similarities in the analysis of Lorenz, Chen, and Lu systems," Applied Mathematics and Computation, vol. 256, pp. 334-343, 2015.

[35] Z. Wei, W. Zhang, Z. Wang, and M. Yao, "Hidden attractors and dynamical behaviors in an extended Rikitake system," International Journal of Bifurcation and Chaos, vol. 25, no. 2, Article ID 1550028, 2015.

[36] Z. C. Wei, I. Moroz, J. C. Sprott, A. Akgul, and W. Zhang, "Hidden hyperchaos and electronic circuit application in a 5D self-exciting homopolar disc dynamo," Chaos, vol. 27, no. 3, Article ID 033101, 2017.

[37] V.-T. Pham, C. Volos, S. Jafari, S. Vaidyanathan, T. Kapitaniak, and X. Wang, "A chaotic system with different families of hidden attractors," International Journal of Bifurcation and Chaos, vol. 26, no. 8, Article ID 1650139, 2016.

[38] V. T. Pham, C. Volos, S. Jafari, and T. Kapitaniak, "Coexistence of hidden chaotic attractors in a novel no-equilibrium system," Nonlinear Dynamics, vol. 87, no. 3, pp. 2001-2010, 2017.

[39] M.-F. Danca, N. Kuznetsov, and G. Chen, "Unusual dynamics and hidden attractors of the Rabinovich-Fabrikant system," Nonlinear Dynamics, vol. 88, no. 1, pp. 791-805, 2017.

[40] G. A. Leonov and N. V. Kuznetsov, "Hidden attractors in dynamical systems. From hidden oscillations in HilbertKolmogorov, aizerman, and kalman problems to hidden chaotic attractor in chua circuits," International Journal of Bifurcation and Chaos, vol. 23, no. 1, Article ID 1330002, 2013.

[41] C. Li and J. C. Sprott, "Coexisting hidden attractors in a 4-D simplified Lorenz system," International Journal of Bifurcation and Chaos, vol. 24, no. 3, p. 1450034, 2014.

[42] A. Wolf, J. B. Swift, H. L. Swinney, and J. A. Vastano, "Determining lyapunov exponents from a time series," Physica $D$ : Nonlinear Phenomena, vol. 16, no. 3, pp. 285-317, 1985.

[43] N. V. Kuznetsov, G. A. Leonov, T. N. Mokaev, A. Prasad, and M. D. Shrimali, "Finite-time Lyapunov dimension and hidden attractor of the Rabinovich system," Nonlinear Dynamics, vol. 92, no. 2, pp. 267-285, 2018.

[44] G. A. Leonov, N. V. Kuznetsov, and T. N. Mokaev, "Homoclinic orbits, and self-excited and hidden attractors in a Lorenz-like system describing convective fluid motion," The European Physical Journal Special Topics, vol. 224, no. 8, pp. 1421-1458, 2015.

[45] B. Zhang, Y. Jia, and J. Du, "Adaptive synchronization control of networked robot systems without velocity measurements," International Journal of Robust and Nonlinear Control, vol. 28, no. 11, pp. 3606-3622, 2018.

[46] S. Vaidyanathan and C. Volos, "Analysis and adaptive control of a novel 3-D conservative no-equilibrium chaotic system," Archives of Control Sciences, vol. 25, no. 3, pp. 333-353, 2015.

[47] H. B. Fotsin and J. Daafouz, "Adaptive synchronization of uncertain chaotic colpitts oscillators based on parameter 
identification," Physics Letters A, vol. 339, no. 3-5, pp. 304-315, 2005.

[48] T.-L. Liao and S.-H. Tsai, "Adaptive synchronization of chaotic systems and its application to secure communications," Chaos, Solitons \& Fractals, vol. 11, no. 9, pp. 1387-1396, 2000.

[49] S. Chen and J. Lü, "Synchronization of an uncertain unified chaotic system via adaptive control," Chaos, Solitons \& Fractals, vol. 14, no. 4, pp. 643-647, 2002.

[50] O. Mofid and S. Mobayen, "Adaptive synchronization of fractional-order quadratic chaotic flows with nonhyperbolic equilibrium," Journal of Vibration and Control, vol. 24, no. 21, pp. 4971-4987, 2018. 